\title{
Commutators of Multilinear Calderón-Zygmund Operator on Weighted Herz-Morrey Spaces with Variable Exponents
}

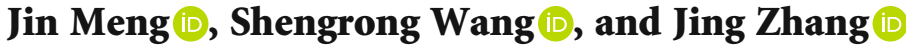 \\ School of Mathematics and Statistics, Hainan Normal University, Haikou 571158, China \\ Correspondence should be addressed to Jing Zhang; zh_jing0820@hotmail.com
}

Received 24 March 2021; Accepted 5 June 2021; Published 29 July 2021

Academic Editor: Maria Alessandra Ragusa

Copyright ( 2021 Jin Meng et al. This is an open access article distributed under the Creative Commons Attribution License, which permits unrestricted use, distribution, and reproduction in any medium, provided the original work is properly cited.

In this paper, we acquire the boundedness of commutators generated by multilinear Calderón-Zygmund operator and BMO functions on products of weighted Herz-Morrey spaces with variable exponents.

\section{Introduction}

The space of all Schwartz functions on $\mathbb{R}^{n}$ was denoted by $\mathcal{S}\left(\mathbb{R}^{n}\right)$, and the space of all tempered distributions on $\mathbb{R}^{n}$ was denoted by $\mathcal{S}^{\prime}\left(\mathbb{R}^{n}\right)$. The space of compactly supported bounded functions denoted by $L_{C}^{\infty}\left(\mathbb{R}^{n}\right)$, and the support set of function $f$ was denoted by $\operatorname{supp}(f)$. On the $m$-fold of the Schwartz function space $\delta\left(\mathbb{R}^{n}\right)$, we also set $T$ as an $m$ -linear operator originally defined and $m \geq 2$, and its value belongs to $\mathcal{S}^{\prime}\left(\mathbb{R}^{n}\right)$ :

$$
T: \underbrace{\mathcal{S}\left(\mathbb{R}^{n}\right) \times \mathcal{S}\left(\mathbb{R}^{n}\right) \times \cdots \times \mathcal{S}\left(\mathbb{R}^{n}\right)}_{m} \longrightarrow \mathcal{S}^{\prime}\left(\mathbb{R}^{n}\right) .
$$

We say that $T$ is an $m$-linear Calderón-Zygmund operator, if for some $p_{1}, \cdots, p_{m} \in[1, \infty)$, it extends to a bounded multilinear operator from $L^{p_{1}} \times L^{p_{2}} \times \cdots \times L^{p_{m}}$ to $L^{p}$ with $1 /$ $p_{1}+1 / p_{2}+\cdots+1 / p_{m}=1 / p$, and for $f_{1}, f_{2}, \cdots, f_{m} \in L_{C}^{\infty}\left(\mathbb{R}^{n}\right)$, $x \notin \cap \cap_{j=1}^{m} \operatorname{supp}\left(f_{j}\right)$

$$
T\left(f_{1}, f_{2}, \cdots, f_{m}\right)(x):=\int_{\left(\mathbb{R}^{n}\right)^{m}} K\left(x, y_{1}, y_{2}, \cdots, y_{m}\right) \prod_{i=1}^{m} f_{i}\left(y_{i}\right) d y_{1} d y_{2} \cdots d y_{m},
$$

where kernel $K$ is a function in $\left(\mathbb{R}^{n}\right)^{m+1}$ away from the diagonal $x=y_{1}=y_{2}=\cdots=y_{m}$ and there exist positive constants $\varepsilon, A$ satisfies the following:

$$
\begin{aligned}
& \left|K\left(x, y_{1}, \cdots, y_{m}\right)\right| \leq A\left(\sum_{i=1}^{m}\left|x-y_{i}\right|\right)^{-m n}, \\
& \left|K\left(x, y_{1}, \cdots, y_{m}\right)-K\left(x^{\prime}, y_{1}, \cdots, y_{m}\right)\right| \leq \frac{A\left|x-x^{\prime}\right|^{\varepsilon}}{\left(\sum_{i=1}^{m}\left|x-y_{i}\right|\right)^{m n+\varepsilon}},
\end{aligned}
$$

whenever $\left|x-x^{\prime}\right| \leq 1 / 2 \max \left\{\left|x-y_{1}\right|,\left|x-y_{2}\right|, \cdots,\left|x-y_{m}\right|\right\}$, and for all $1 \leq i \leq m$,

$$
\begin{aligned}
& \left|K\left(x, y_{1}, \cdots, y_{i}, \cdots, y_{m}\right)\right|-\left|K\left(x, y_{1}, \cdots, y_{i}^{\prime}, \cdots, y_{m}\right)\right| \\
& \quad \leq \frac{A\left|y_{i}-y_{i}^{\prime}\right|^{\varepsilon}}{\left(\sum_{i=1}^{m}\left|x-y_{i}\right|\right)^{m n+\varepsilon}},
\end{aligned}
$$

where $\left|y_{i}-y_{i}^{\prime}\right| \leq 1 / 2 \max \left\{\left|x-y_{1}\right|,\left|x-y_{2}\right|, \cdots,\left|x-y_{m}\right|\right\}$.

If $b \in L_{\mathrm{loc}}^{1}\left(\mathbb{R}^{n}\right)$, set

$$
\|b\|_{*}:=\sup _{B} \frac{1}{|B|} \int_{B}\left|b(x)-b_{B}\right| d x
$$

where $b_{B}=(1 /|B|) \int_{B} b(y) d y$ and the supremum is taken over all $B \subset \mathbb{R}^{n}$, and what follows $|B|$ is the Lebesgue measure of measurable set $B$ in $\mathbb{R}^{n}$. A function $b$ is called bounded mean 
oscillation if $\|b\|_{*}<\infty$. Denote by $\mathrm{B} M O\left(\mathbb{R}^{n}\right)$ the set of all bounded mean oscillation functions on $\mathbb{R}^{n}$.

Although our method suits any multilinear operator, only the bilinear Calderón-Zygmund operator will be considered here for the sake of simplicity. Specifically, we will discuss the commutator of a bilinear Calderón-Zygmund operator $T$, BMO functions $b_{1}$ and $b_{2}$, and suitable functions $f_{1}$ and $f_{2}$,

$$
\begin{aligned}
{\left[b_{1}, b_{2}, T\right]\left(f_{1}, f_{2}\right)(x)=} & b_{1}(x) b_{2}(x) T\left(f_{1}, f_{2}\right)(x) \\
& -b_{1}(x) T\left(f_{1}, b_{2} f_{2}\right)(x) \\
& -b_{2}(x) T\left(b_{1} f_{1}, f_{2}\right)(x) \\
& +T\left(b_{1} f_{1}, b_{2} f_{2}\right) .
\end{aligned}
$$

Many analyses of linear commutators have been extended to other fields, such as weighted space, homogeneous space, multiparameter, and multilinear settings. Huang and $\mathrm{Xu}$ [1] obtained boundedness of multilinear singular integrals and their commutators from products of variable exponent Lebesgue spaces to variable exponent Lebesgue spaces. Huet al. [2] proved the boundedness of commutators generated by fractional integrals and BMO on generalized Herz spaces with general Muckenhoupt weights. Tang et al. [3] obtained the boundedness of a commutator generated by the multilinear Calderón-Zygmund operator and $\mathrm{BMO}$ functions in Herz-Morrey spaces with variable exponents. Chen et al. [4] studied multiple weighted norm inequalities for maximal vector-valued multilinear singular operator and maximal commutators. Wang et al. [5] proved the boundedness for a class of multisublinear singular integral operators on the product central Morrey spaces with variable exponents.

Motivated by the mentioned works, we will consider the boundedness of commutators generated by multilinear Calderón-Zygmund operator and BMO functions on products of weighted Herz-Morrey spaces with variable exponents.

\section{Notations and Main Result}

In this section, we recall some notations and definitions; then, we describe our results. Assume $p(\cdot)$ be a measurable function on $\mathbb{R}^{n}$ and take values in $[1, \infty)$, the Lebesgue space with variable exopnent $L^{p(\cdot)}\left(\mathbb{R}^{n}\right)$ is acquired by

$$
\begin{aligned}
L^{p(\cdot)}\left(\mathbb{R}^{n}\right) & :=\left\{f \text { is measurable }: \int_{\mathbb{R}^{n}}\left(\frac{|f(x)|}{\lambda}\right)^{p(x)} d x\right. \\
& <\infty \text { ofor some } \lambda>0\} .
\end{aligned}
$$

The norm is defined by

$$
\|f\|_{L^{p()}}:=\inf \left\{\lambda>0: \int_{\mathbb{R}^{n}}\left(\frac{|f(x)|}{\lambda}\right)^{p(x)} d x \leq 1\right\} .
$$

On a Banach function space, the Lebesgue space $L^{p(\cdot)}\left(\mathbb{R}^{n}\right)$ is equipped with the norm $\|f\|_{L^{p^{(\cdot)}}}$ The space $L_{\text {loc }}^{p^{(\cdot)}}\left(\mathbb{R}^{n}\right)$ is defined by

$L_{\text {loc }}^{p(\cdot)}\left(\mathbb{R}^{n}\right):=\left\{f: f_{\chi_{K}} \in L^{p(\cdot)}\left(\mathbb{R}^{n}\right)\right.$ for all compact subsets $\left.K \subset \mathbb{R}^{n}\right\}$,

where and what follows, $\chi_{A}$ denotes the characteristic function of a measurable set $A \subset \mathbb{R}^{n}$.

Let $p(\cdot): \mathbb{R}^{n} \longrightarrow(0, \infty)$, we denote

$$
p_{-}:=\operatorname{ess} \inf _{x \in \mathbb{R}^{n}} p(x), p_{+}:=\operatorname{ess} \sup _{x \in \mathbb{R}^{n}} p(x)
$$

The set $\mathscr{P}\left(\mathbb{R}^{n}\right)$ consists of all $p(\cdot)$ satisfying $p_{-}>1$ and $p_{+}$ $<\infty ; \mathscr{P}_{0}\left(\mathbb{R}^{n}\right)$ consists of all $p(\cdot)$ satisfying $p_{-}>0$ and $p_{+}<$ $\infty$. $L^{p(\cdot)}$ can be equally defined as above for $p(\cdot) \in \mathscr{P}_{0}\left(\mathbb{R}^{n}\right) \cdot q^{\prime}$ $(\cdot)$ is the conjugate exponent of $p(\cdot)$, defined pointwise by $1 / p$ $(\cdot)+1 / p^{\prime}(\cdot)=1$.

Let $p(\cdot) \in \mathscr{P}\left(\mathbb{R}^{n}\right)$ and $w$ be a weight which is a nonnegative measurable function on $\mathbb{R}^{n}$. Then, the weighted variable exponent Lebesgue space $L^{p(\cdot)}(w)$ is the set of all complexvalued measurable function $f$ such that $f w \in L^{p(\cdot)}$. The space $L^{p(\cdot)}(w)$ is a Banach space equipped with the norm

$$
\|f\|_{L^{p^{(\cdot)}}(w)}:=\|f w\|_{L^{p^{(\cdot)}}} .
$$

Let $f \in L_{\text {loc }}^{1}\left(\mathbb{R}^{n}\right)$. Then, the standard Hardy-Littlewood maximal function of $f$ is defined by

$$
M f(x):=\sup _{B \ni x} \frac{1}{|B|} \int_{B}|f(y)| d y, \forall x \in \mathbb{R}^{n},
$$

where the supremum is taken over all balls containing $x$ in $\mathbb{R}^{n}$. Generally speaking, on weighted variable Lebesgue spaces, the Hardy-Littlewood maximal operator is not bounded. But if it meets certain conditions, it will be established. Namely, let $p(\cdot) \in \mathscr{P}\left(\mathbb{R}^{n}\right)$ and meet the following global $\log$-Hölder continuous and $w \in A_{p(\cdot)}$ such that $M$ is bounded on $L^{p(\cdot)}(\mathrm{w})$, see [6].

Definition 1. Assume $\alpha(\cdot)$ be a real-valued measurable function on $\mathbb{R}^{n}$.

(i) We say that $\alpha(\cdot)$ satisfies the local log-Hölder continuity condition if there exists a constant $C_{1}$ such that

$|\alpha(x)-\alpha(y)| \leq \frac{C_{1}}{\log (e+(1 /|x-y|))}, x, y \hat{\mathrm{G}} \in \mathbb{R}^{n},|x-y|<\frac{1}{2}$ 
(ii) We say that $\alpha(\cdot)$ satisfies the log-Hölder continuous at the origin if there exists a constant $C_{2}$ such that

$$
|\alpha(x)-\alpha(0)| \leq \frac{C_{2}}{\log (e+(1 /|x|))}, \forall x \in \mathbb{R}^{n}
$$

Denote by $\mathscr{P}_{0}^{\log }\left(\mathbb{R}^{n}\right)$ the set of all log-Hölder continuous functions at the origin.

(iii) We say that $\alpha(\cdot)$ satisfies the log-Hölder continuous at the infinity if there exists $\alpha_{\infty} \in \mathbb{R}$ and a constant $C_{3}$ such that

$$
\left|\alpha(x)-\alpha_{\infty}\right| \leq \frac{C_{3}}{\log (e+|x|)}, \forall x \in \mathbb{R}^{n}
$$

Denote by $\mathscr{P}_{\infty}^{\log }\left(\mathbb{R}^{n}\right)$ the set of all log-Hölder continuous functions at infinity.

(iv) We say that $\alpha(\cdot)$ satisfies the global log-Hölder continuous if $\alpha(\cdot)$ is both log-Hölder continuous and locally log-Hölder continuous at infinity. We denote by $\mathscr{P}^{\log }\left(\mathbb{R}^{n}\right)$ the set of all global log-Hölder continuous functions

Definition 2. Given $p(\cdot) \in \mathscr{P}\left(\mathbb{R}^{n}\right)$ and a positive measurable function $w$, we say that $w \in A_{p(\cdot)}$ if there exists a positive constant $C$ for all balls $B$ in $\mathbb{R}^{n}$ such that

$$
\frac{1}{|B|}\left\|w \chi_{B}\right\|_{L^{p(\cdot)}}\left\|w^{-1} \chi_{B}\right\|_{L^{p^{\prime}(\cdot)}} \leq C .
$$

Remark 3. In [7], Cruz-Uribe et al. obtained that if $p(\cdot) \in \mathscr{P}\left(\mathbb{R}^{n}\right)$ and $w \in A_{p(\cdot)}$, then $w^{-1} \in A_{p^{\prime}(\cdot)}$.

The Muckenhoupt $A_{p}$ class with constant exponent $p \in(1, \infty)$ was firstly proposed by Muckenhoupt in [8]. The variable Muckenhoupt $A_{p(\cdot)}$ was considered in [7, 9-12].

Lemma 4 (see [7, Theorem 1.5]). If $p(\cdot) \in \mathscr{P}^{\log }\left(\mathbb{R}^{n}\right) \cap \mathscr{P}\left(\mathbb{R}^{n}\right)$ and $w \in A_{p(\cdot)}$, then there is a positive constant $C$ such that for each $f \in L^{p(\cdot)}(w)$,

$$
\|(M f) w\|_{L^{p(\cdot)}} \leq C\|f w\|_{L^{p(\cdot)}}
$$

Next, we define the weighted Herz-Morrey space with variable exponents, and we use the following concepts. Let $k \in \mathbb{Z}$, we define

$$
\begin{aligned}
B_{k} & :=\left\{x \in \mathbb{R}^{n}:|x| \leq 2^{k}\right\}, \\
D_{k} & :=B_{k} \backslash B_{k-1}, \\
\chi_{k} & :=\chi_{D_{k}}, \\
\tilde{\chi}_{m} & =\chi_{m}, m \geq 1, \\
\tilde{\chi}_{0} & =\chi_{B_{0}} .
\end{aligned}
$$

Definition 5. Let $q(\cdot), p(\cdot) \in \mathscr{P}_{0}\left(\mathbb{R}^{n}\right), \lambda \in[0, \infty)$. Let $\alpha(\cdot)$ be a bounded real-valued measurable function on $\mathbb{R}^{n}$. The nonhomogeneous weighted Herz-Morrey space $M K_{q(\cdot), \lambda}^{\alpha(\cdot), p(\cdot)}(w)$ and homogeneous weighted Herz-Morrey space $M \dot{K}_{q(\cdot), \lambda}^{\alpha(\cdot), p(\cdot)}(w)$ are defined, respectively, by

$$
\begin{aligned}
M K_{q(\cdot), \lambda}^{\alpha(\cdot), p(\cdot)}(w): & =\left\{f \in L_{\mathrm{loc}}^{p(\cdot)}\left(\mathbb{R}^{n}, w\right):\|f\|_{M K_{q(\cdot), \lambda}^{\alpha(\cdot), p(\cdot)}(w)}<\infty\right\}, \\
M \dot{K}_{q(\cdot), \lambda}^{\alpha(\cdot), p(\cdot)}(w): & =\left\{f \in L_{\mathrm{loc}}^{p(\cdot)}\left(\mathbb{R}^{n} \backslash\{0\}, w\right):\|f\|_{M \dot{K}_{q(\cdot), \lambda}^{\alpha(\cdot), p(\cdot)}(w)}<\infty\right\},
\end{aligned}
$$

and where

$$
\begin{aligned}
& \|f\|_{M \dot{K}_{q(\cdot), \lambda}^{\alpha(\cdot) p(\cdot)}(w)}:=\sup _{L \in \mathbb{Z}} 2^{-L \lambda}\left\|\left(2^{\alpha(\cdot) k} f \chi_{k}\right)_{k \leq L}\right\|_{\ell^{q(\cdot)}\left(L^{p(\cdot)}(w)\right)}, \\
& \|f\|_{M K_{q(\cdot), \lambda}^{\alpha(\cdot), \cdot(\cdot)}(w)}:=\sup _{L \in \mathbb{N}_{0}} 2^{-L \lambda}\left\|\left(2^{\alpha(\cdot) k} f \tilde{\chi}_{k}\right)_{k=0}^{L}\right\|_{\ell^{q(\cdot)}\left(L^{p(\cdot)}(w)\right)} .
\end{aligned}
$$

Let $B$ and $C$ be two real numbers. If there exists a constant $K>0$ such that $B \leq K C$, we denote $B \lesssim C$. If $B \lesssim C$ and $C \lesssim B$, we denote $B \approx C$.

Proposition 6 (see [13, Proposition 1]). Let $p(\cdot), q(\cdot) \in \mathscr{P}_{0}$ $\left(\mathbb{R}^{n}\right)$, w be a weight, $\lambda \in[0, \infty)$, and $\alpha(\cdot) \in L^{\infty}\left(\mathbb{R}^{n}\right)$.

(i) If $\alpha(\cdot), q(\cdot) \in \mathscr{P}_{0}^{\log }\left(\mathbb{R}^{n}\right) \cap \mathscr{P}_{\infty}^{\log }\left(\mathbb{R}^{n}\right)$, then for all $f \in$ $L_{l o c}^{p(\cdot)}\left(\mathbb{R}^{n} \backslash\{0\}, w\right)$

$$
\begin{aligned}
& \|f\|_{M \dot{K}_{P(\cdot), \lambda}^{\alpha(\cdot),()}(w)} \approx \max \left\{\sup _{L \leq 0, L \in \mathbb{Z}} 2^{-L \lambda}\left\|\left(2^{k \alpha(0)} f \chi_{k}\right)_{k \leq L}\right\|_{\ell^{g_{0}}\left(L^{p(\cdot)}(w)\right)} \sup _{L>0, L \in \mathbb{Z}}\right. \\
& \cdot\left[2^{-L \lambda}\left\|\left(2^{k \alpha(0)} f \chi_{k}\right)_{k<0}\right\|_{\ell^{q_{0}}\left(L^{(\cdot)}(w)\right)}\right. \\
& \left.\left.+2^{-L \lambda}\left\|\left(2^{k \alpha_{\infty}} f \chi_{k}\right)_{k=0}^{L}\right\|_{e^{q \infty}\left(L^{p())}(w)\right)}\right]\right\} \text {, }
\end{aligned}
$$

where and hereafter, $q_{0}:=q(0)$

(ii) If $\alpha(\cdot), q(\cdot) \in \mathscr{P}_{\infty}^{\log }\left(\mathbb{R}^{n}\right)$, then 


$$
M K_{p(\cdot), \lambda}^{\alpha(\cdot), q(\cdot)}(w)=M K_{p(\cdot), \lambda}^{\alpha_{\infty}, q_{\infty}}(w)
$$

Lemma 7 has been proved by Noi and Izuki in $[14,15]$.

Lemma 7. If $p(\cdot) \in \mathscr{P}^{\log }\left(\mathbb{R}^{n}\right) \cap \mathscr{P}\left(\mathbb{R}^{n}\right)$ and $w \in A_{p(\cdot)}$, then there exist constants $\delta_{1}, \delta_{2} \in(0,1)$ and $C>0$ such that for all balls $B$ in $\mathbb{R}^{n}$ and all measurable subsets $S \subset B$,

$$
\begin{gathered}
\frac{\left\|\chi_{S}\right\|_{L^{p(\cdot)}(w)}}{\left\|\chi_{B}\right\|_{L^{p(\cdot)}(w)}} \leq C\left(\frac{|S|}{|B|}\right)^{\delta_{1}}, \\
\frac{\left\|\chi_{S}\right\|_{L^{p^{\prime}(\cdot)}\left(w^{-1}\right)}}{\left\|\chi_{B}\right\|_{L^{p^{\prime}(\cdot)}\left(w^{-1}\right)}} \leq C\left(\frac{|S|}{|B|}\right)^{\delta_{2}} .
\end{gathered}
$$

The following is the main result.

Theorem 8. Let $T$ be a bilinear Calderón-Zygmund operator and let $b_{1}$ and $b_{2}$ be BMO functions. Given $p_{1}(\cdot)$, $p_{2}(\cdot) \in \mathscr{P}^{\log }\left(\mathbb{R}^{n}\right) \cap \mathscr{P}\left(\mathbb{R}^{n}\right)$ satisfying $1 / p(x)=\left(1 / p_{1}(x)\right)+(1 /$ $\left.p_{2}(x)\right)$ for $x \in \mathbb{R}^{n}$. Let $w_{1}, w_{2}$ be weights, $w=w_{1} w_{2}, w_{i} \in A_{p_{i}(\cdot)}$, $i=1$, 2. Assume that $\alpha(\cdot) \in L^{\infty}\left(\mathbb{R}^{n}\right) \cap \mathscr{P}_{0}^{\log }\left(\mathbb{R}^{n}\right) \cap \mathscr{P}_{\infty}^{\log }\left(\mathbb{R}^{n}\right)$, $\alpha(0)=\alpha_{1}(0)+\alpha_{2}(0), \quad \alpha_{\infty}=\alpha_{1 \infty}+\alpha_{2 \infty}, \quad q(\cdot) \in \mathscr{P}_{0}^{\log }\left(\mathbb{R}^{n}\right) \cap$ $\mathscr{P}_{\infty}^{\log }\left(\mathbb{R}^{n}\right), 1 / q(0)=1 / q_{1}(0)+1 / q_{2}(0), \quad 1 / q_{\infty}=1 / q_{1 \infty}+1 / q_{2 \infty}$, $\lambda=\lambda_{1}+\lambda_{2}, 0 \leq \lambda_{i}<\infty, \delta_{i 1}, \delta_{i 2} \in(0,1)$ are the constants in Lemma 7 for exponents $p_{i}(\cdot)$ and weights $w_{i}, i=1,2$. If $\lambda_{i}-n$ $\delta_{i 1}<\alpha_{i \infty}, \alpha_{i}(0)<n \delta_{i 2}, i=1,2$, then

$$
\left\|\left[b_{1}, b_{2}, T\right]\left(f_{1}, f_{2}\right)\right\|_{M \dot{K}_{p(\cdot), \lambda}^{\alpha(\cdot), q(\cdot)}(w)} \leqslant\left\|f_{1}\right\|_{M \dot{K}_{p_{1}(\cdot), \lambda_{1}}^{\alpha_{1}(\cdot), q_{1}(\cdot)}\left(w_{1}\right)}\left\|f_{2}\right\|_{M \dot{K}_{p_{2}(\cdot), \lambda_{2}}^{\alpha_{2}(\cdot), q_{2}(\cdot)}\left(w_{2}\right)} .
$$

\section{Proof of Theorem 8}

Before we prove Theorem 8, we need to introduce some lemmas.

Lemma 9 (see $\left[1\right.$, Theorem 2.3]). Let $p(\cdot), p_{1}(\cdot), p_{2}(\cdot) \in \mathscr{P}_{0}$ $\left(\mathbb{R}^{n}\right)$ such that $1 / p(x)=1 / p_{1}(x)+1 / p_{2}(x)$ for $x \in \mathbb{R}^{n}$. Then, there exists a constant $C_{p, p_{1}}$ independent of functions $f$ and $g$ such that

$$
\|f g\|_{L^{p(\cdot)}} \leq C_{p, p_{1}}\|f\|_{L^{p_{1}(\cdot)}}\|g\|_{L^{p_{2}(\cdot)}},
$$

holds for every $f \in L^{p_{1}(\cdot)}\left(\mathbb{R}^{n}\right)$ and $g \in L^{p_{2}(\cdot)}\left(\mathbb{R}^{n}\right)$.

If $p \in \mathscr{P}\left(\mathbb{R}^{n}\right), w \in A_{p(\cdot)}$ with $w=w_{1} w_{2}$, then by the Hölder inequality, we have

$$
\|f g\|_{L^{p(\cdot)}(w)} \leq C_{p, p_{1}}\|f\|_{L^{p_{1}(\cdot)}\left(w_{1}\right)}\|g\|_{L^{p_{2}(\cdot)}\left(w_{2}\right)} .
$$

Lemma 10 (see [16, Corollary 3.11]). Let $b \in B M O\left(\mathbb{R}^{n}\right)$, $p(\cdot)$ $\in \mathscr{P}^{\log }\left(\mathbb{R}^{n}\right) \cap \mathscr{P}\left(\mathbb{R}^{n}\right), w \in A_{p(\cdot)}, t \in[1, \infty)$, and $k, i \in \mathbb{N}$ such that $k>i$, then one has

$$
\left\|\left|b-b_{B_{i}}\right|^{t} \chi_{B_{k}}\right\|_{L^{p(\cdot)}(w)} \leq C(k-i)^{t}\|b\|_{*}^{t}\left\|\chi_{B_{k}}\right\|_{L^{p(\cdot)}(w)} .
$$

Lemma 11 (see [17, Theorem 2.6]). If $0<p<\infty$ and $\delta>0$, then there exists a positive constant $C$ such that

$$
\left(\sum_{l=-\infty}^{\infty}\left(\sum_{k=-\infty}^{\infty} 2^{-|k-l| \delta} a_{k}\right)^{p}\right)^{1 / p} \leq C\left(\sum_{l=-\infty}^{\infty} a_{l}^{p}\right)^{1 / p}
$$

for nonnegative sequences $\left\{a_{l}\right\}_{l=-\infty}^{\infty}$.

Lemma 12 (see [10, Theorems 2.23 and 2.24]). Assume that for some $p_{0}, p_{0} \in(1, \infty)$, and every $w_{0} \in A_{\infty}$,

$$
\int_{\mathbb{R}^{n}} f(x)^{p_{0}} w_{0}(x) d x \leq C \int_{\mathbb{R}^{n}} g(x)^{p_{0}} w_{0}(x) d x,(f, g) \in \mathscr{F}
$$

where $\mathscr{F}$ is a pair of nonnegative functions. Given $p(\cdot) \in \mathscr{P}_{0}($ $\left.\mathbb{R}^{n}\right)$, assume that there exists $s \leq p_{-}$such that $w^{s} \in A_{p(\cdot) / s}$ and $M$ is bounded on $L^{(p(\cdot) / s)^{\prime}}\left(w^{-s}\right)\left(\mathbb{R}^{n}\right)$. Then,

$$
\|f\|_{L^{p(\cdot)}(w)} \leq C\|g\|_{L^{p(\cdot)}(w)},(f, g) \in \mathscr{F}
$$

Lemma 13. Let $p_{1}(\cdot), p_{2}(\cdot) \in \mathscr{P}\left(\mathbb{R}^{n}\right), 1<\left(p_{i}\right)_{-} \leq\left(p_{i}\right)_{+}<\infty$, and $p_{i}(\cdot) \in \mathscr{P}^{\log }\left(\mathbb{R}^{n}\right) \cap \mathscr{P}\left(\mathbb{R}^{n}\right)$ satisfying $1 / p(x)=1 / p_{1}(x)+1$ $/ p_{2}(x)$ for $x \in \mathbb{R}^{n}, i=1,2$. Let $b_{1}$ and $b_{2}$ be BMO functions. Let $w_{1} \in A_{p_{1}(\cdot)}, w_{2} \in A_{p_{2}(\cdot)}$, and $w=w_{1} w_{2}$. If $T$ is a bilinear Calderón-Zygmund operator, then

$$
\left\|\left[b_{1}, b_{2}, T\right]\left(f_{1}, f_{2}\right)\right\|_{L^{p(\cdot)}(w)} \leqslant\left\|f_{1}\right\|_{L^{p_{1}(\cdot)}\left(w_{1}\right)}\left\|f_{2}\right\|_{L^{p^{2}(\cdot)}\left(w_{2}\right)} \cdot
$$

Proof. We assume that $b_{1}$ and $b_{2}$ are bounded functions. Let $f_{1}$ and $f_{2}$ be bounded functions with compact support. By the same argument in the proof of [1, Theorem 2.2], we have

$$
\begin{aligned}
& \int_{\mathbb{R}^{n}}\left|\left[b_{1}, b_{2}, T\right]\left(f_{1}, f_{2}\right)(x)\right|^{p_{0}} w_{0}(x) d x \\
& \quad \leq\left\|b_{1}\right\|_{*}^{p_{0}}\left\|b_{2}\right\|_{*}^{p_{0}} \int_{\mathbb{R}^{n}}\left(M_{1} f_{1}(x) M_{2} f_{2}(x)\right)^{p_{0}} w_{0}(x) d x .
\end{aligned}
$$

By Lemma 12, we have

$$
\left\|\left[b_{1}, b_{2}, T\right]\left(f_{1}, f_{2}\right)\right\|_{L^{p(\cdot)}(w)} \leqslant\left\|b_{1}\right\|_{*}\left\|b_{2}\right\|_{*}\left\|M_{1} f_{1} M_{2} f_{2}\right\|_{L^{p(\cdot)}(w)} .
$$

By Lemmas 9 and 4, we have

$$
\left\|\left[b_{1}, b_{2}, T\right]\left(f_{1}, f_{2}\right)\right\|_{L^{p(\cdot)}(w)} \leqslant\left\|b_{1}\right\|_{*}\left\|b_{2}\right\|_{*}\left\|f_{1}\right\|_{L^{p_{1}(\cdot)}\left(w_{1}\right)}\left\|f_{2}\right\|_{L^{p^{2}(\cdot)}\left(w_{2}\right)} \cdot
$$


Proof of Theorem 8. Assume $f_{1}$ and $f_{2}$ are bounded functions with compact support and write

$$
f_{i}=\sum_{l=-\infty}^{\infty} f_{i} \chi_{l}=: \sum_{l=-\infty}^{\infty} f_{i l}, i=1,2
$$

By Proposition 6, we have

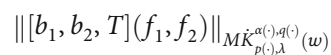

$$
\begin{aligned}
& \approx \max \left\{\sup _{L \leq 0, L \in \mathbb{Z}} 2^{-L \lambda}\left\|\left(2^{k \alpha(0)}\left[b_{1}, b_{2}, T\right]\left(f_{1}, f_{2}\right) \chi_{k}\right)_{k \leq L}\right\|_{\eta^{0}\left(L^{P(\theta)}(w)\right)}, \sup _{L>0, L \in \mathbb{Z}}\right. \\
& \times\left[2^{-L \lambda}\left\|\left(2^{k \alpha(0)}\left[b_{1}, b_{2}, T\right]\left(f_{1}, f_{2}\right) \chi_{k}\right)_{k<0}\right\|_{\left.l^{p_{0}}\left(L^{p()}\right)(w)\right)}\right. \\
& \left.\left.+2^{-L \lambda}\left\|\left(2^{k \alpha_{\infty}}\left[b_{1}, b_{2}, T\right]\left(f_{1}, f_{2}\right) \chi_{k}\right)_{k=0}^{L}\right\|_{\mu^{\rho_{\infty}}\left(L^{\left.()^{()}\right)}(w)\right)}\right]\right\}:=\max \{E, H\},
\end{aligned}
$$

where

$$
\begin{aligned}
& E:=\sup _{L \leq 0, L \in \mathbb{Z}} 2^{-L \lambda}\left\|\left(2^{k \alpha(0)}\left[b_{1}, b_{2}, T\right]\left(f_{1}, f_{2}\right) \chi_{k}\right)_{k \leq L}\right\|_{l^{9_{0}}\left(L^{p(\cdot)}(w)\right)}, \\
& H:=\sup _{L \in \mathbb{N}}\{F+G\}, \\
& F:=2^{-L \lambda}\left\|\left(2^{k \alpha(0)}\left[b_{1}, b_{2}, T\right]\left(f_{1}, f_{2}\right) \chi_{k}\right)_{k<0}\right\|_{l_{q^{0}}\left(L^{p(\cdot)}(w)\right)}, \\
& \mathrm{G}:=2^{-L \lambda}\left\|\left(2^{k \alpha_{\infty}}\left[b_{1}, b_{2}, T\right]\left(f_{1}, f_{2}\right) \chi_{k}\right)_{k=0}^{L}\right\|_{l^{\mu_{\infty}}\left(L^{p(\cdot)}(w)\right)} .
\end{aligned}
$$

Since the estimates of $E$ and $F$ are essentially analogical, we only need to obtain $E$ and $G$ bounded in the HerzMorrey space with variable exponents. It is easy to see that

$$
E \leq C \sum_{i=i}^{9} E_{i}, G \leq C \sum_{i=i}^{9} G_{i}
$$

where

$$
\begin{aligned}
& E_{1}:=\sup _{L \leq 0, L \in \mathbb{Z}} 2^{-L \lambda}\left(\sum_{k=-\infty}^{L} 2^{k \alpha(0) q(0)}\left\|\sum_{l=-\infty}^{k-2} \sum_{j=-\infty}^{k-2}\left[b_{1}, b_{2}, T\right]\left(f_{11}, f_{2 j}\right) \chi_{k}\right\|_{L^{(\theta)}(w)}^{q(0)}\right)^{1 / q(0)}, \\
& E_{2}:=\sup _{L \leq 0, L \in \mathbb{Z}} 2^{-L \lambda}\left(\sum_{k=-\infty}^{L} 2^{k \alpha(0) q(0)}\left\|\sum_{l=-\infty}^{k-2} \sum_{j=k-1}^{k+1}\left[b_{1}, b_{2}, T\right]\left(f_{11}, f_{2 j}\right) \chi_{k}\right\|_{L^{\rho(\theta)}(w)}^{q(0)}\right)^{1 / q(0)}, \\
& E_{3}:=\sup _{L \leq 0, L \in \mathbb{Z}} 2^{-L \lambda}\left(\sum_{k=-\infty}^{L} 2^{k \alpha(0) q(0)}\left\|\sum_{l=-\infty}^{k-2} \sum_{j=k+2}^{\infty}\left[b_{1}, b_{2}, T\right]\left(f_{11}, f_{2 j}\right) \chi_{k}\right\|_{L^{(j)}(w)}^{q(0)}\right)^{1 / q(0)}, \\
& E_{4}:=\sup _{L \leq 0, L \in \mathbb{Z}} 2^{-L \lambda}\left(\sum_{k=-\infty}^{L} 2^{k \alpha(0) q(0)}\left\|\sum_{l=k-1}^{k+1} \sum_{j=-\infty}^{k-2}\left[b_{1}, b_{2}, T\right]\left(f_{1 p}, f_{2 j}\right) x_{k}\right\|_{L^{p^{(*)}(w)}}^{q(0)}\right)^{1 / q(0)}, \\
& E_{5}:=\sup _{L \leq 0, L \in \mathbb{Z}} 2^{-L \lambda}\left(\sum_{k=-\infty}^{L} 2^{k \alpha(0) q(0)}\left\|\sum_{l=k-1}^{k+1} \sum_{j=k-1}^{k+1}\left[b_{1}, b_{2}, T\right]\left(f_{1}, f_{2 j}\right) \chi_{k}\right\|_{L^{p(\theta)}(w)}^{q(0)}\right)^{1 / q(0)} \text {, }
\end{aligned}
$$

$$
\begin{aligned}
& E_{6}:=\sup _{L \leq 0, L \in \mathbb{Z}} 2^{-L \lambda}\left(\sum_{k=-\infty}^{L} 2^{k \alpha(0) q(0)}\left\|\sum_{l=k-1}^{k+1} \sum_{j=k+2}^{\infty}\left[b_{1}, b_{2}, T\right]\left(f_{11}, f_{2 j}\right) \chi_{k}\right\|_{L^{\ell()}(w)}^{q(0)}\right)^{1 / q(0)}, \\
& E_{7}:=\sup _{L \leq 0, L \in \mathbb{Z}} 2^{-L \lambda}\left(\sum_{k=-\infty}^{L} 2^{k \alpha(0) q(0)}\left\|\sum_{l=k+2}^{\infty} \sum_{j=-\infty}^{k-2}\left[b_{1}, b_{2}, T\right]\left(\mathrm{f}_{11}, f_{2 j}\right) \chi_{k}\right\|_{L^{\ominus}()(w)}^{q(0)}\right)^{1 / q(0)}, \\
& E_{8}:=\sup _{L \leq 0, L \in \mathbb{Z}} 2^{-L \lambda}\left(\sum_{k=-\infty}^{L} 2^{k \alpha(0) q(0)}\left\|\sum_{l=k+2}^{\infty} \sum_{j=k-1}^{k+1}\left[b_{1}, b_{2}, T\right]\left(f_{1 l}, f_{2 j}\right) \chi_{k}\right\|_{L^{(\theta)}(w)}^{q(0)}\right)^{1 / q(0)}, \\
& E_{9}:=\sup _{L \leq 0, L \in \mathbb{Z}} 2^{-L \lambda}\left(\sum_{k=-\infty}^{L} 2^{k \alpha(0) q(0)}\left\|\sum_{l=k+2}^{\infty} \sum_{j=k+2}^{\infty}\left[b_{1}, b_{2}, T\right]\left(f_{11}, f_{2 j}\right) \chi_{k}\right\|_{L^{(\ell)}(w)}^{q(0)}\right)^{1 / q(0)}, \\
& G_{1}:=2^{-L \lambda}\left(\sum_{k=0}^{L} 2^{k \alpha_{\infty} q_{\infty}}\left\|\sum_{l=-\infty}^{k-2} \sum_{j=-\infty}^{k-2}\left[b_{1}, b_{2}, T\right]\left(f_{1 p}, f_{2 j}\right) \chi_{k}\right\|_{L^{p(\theta)}(w)}^{q_{\infty}}\right)^{1 / q_{\infty}}, \\
& G_{2}:=2^{-L \lambda}\left(\sum_{k=0}^{L} 2^{k k_{\infty} q_{\infty}}\left\|\sum_{l=-\infty}^{k-2} \sum_{j=k-1}^{k+1}\left[b_{1}, b_{2}, T\right]\left(f_{1}, f_{2 j}\right) \chi_{k}\right\|_{L^{\rho(\theta)}(w)}^{q_{\infty}}\right)^{1 / q_{\infty}}, \\
& G_{3}:=2^{-L \lambda}\left(\sum_{k=0}^{L} 2^{k \alpha_{\infty} q_{\infty}}\left\|\sum_{l=-\infty}^{k-2} \sum_{j=k+2}^{\infty}\left[b_{1}, b_{2}, T\right]\left(f_{11}, f_{2 j}\right) \chi_{k}\right\|_{L^{\rho(\ell)}(w)}^{q_{\infty}}\right)^{1 / q_{\infty}}, \\
& G_{4}:=2^{-L \lambda}\left(\sum_{k=0}^{L} 2^{k \alpha_{\infty} q_{\infty}}\left\|\sum_{l=k-1}^{k+1} \sum_{j=-\infty}^{k-2}\left[b_{1}, b_{2}, T\right]\left(f_{11}, f_{2 j}\right) x_{k}\right\|_{L^{\beta(\gamma)}(w)}^{q_{\infty}}\right)^{1 / q_{\infty}}, \\
& G_{5}:=2^{-L \lambda}\left(\sum_{k=0}^{L} 2^{k \alpha_{\infty} q_{\infty}}\left\|\sum_{l=k-1}^{k+1} \sum_{j=k-1}^{k+1}\left[b_{1}, b_{2}, T\right]\left(f_{11}, f_{2 j}\right) \chi_{k}\right\|_{L^{p())}(w)}^{q_{\infty}}\right)^{1 / q_{\infty}}, \\
& G_{6}:=2^{-L \lambda}\left(\sum_{k=0}^{L} 2^{k \alpha_{\infty} q_{\infty}}\left\|\sum_{l=k-1}^{k+1} \sum_{j=k+2}^{\infty}\left[b_{1}, b_{2}, T\right]\left(f_{1 l}, f_{2 j}\right) \chi_{k}\right\|_{L^{p()}(w)}^{q_{\infty}}\right)^{1 / q_{\infty}}, \\
& G_{7}:=2^{-L \lambda}\left(\sum_{k=0}^{L} 2^{k k_{\infty} q_{\infty}}\left\|\sum_{l=k+2}^{\infty} \sum_{j=-\infty}^{k-2}\left[b_{1}, b_{2}, T\right]\left(f_{11}, f_{2 j}\right) \chi_{k}\right\|_{L^{(\ell)}(w)}^{q_{\infty}}\right)^{1 / q_{\infty}}, \\
& G_{8}:=2^{-L \lambda}\left(\sum_{k=0}^{L} 2^{k \alpha_{\infty} q_{\infty}}\left\|\sum_{l=k+2}^{\infty} \sum_{j=k-1}^{k+1}\left[b_{1}, b_{2}, T\right]\left(f_{1}, f_{2 j}\right) \chi_{k}\right\|_{L^{(\ell)}(w)}^{q_{\infty}}\right)^{1 / q_{\infty}}, \\
& G_{9}:=2^{-L \lambda}\left(\sum_{k=0}^{L} 2^{k \alpha_{\infty} q_{\infty}}\left\|\sum_{l=k+2}^{\infty} \sum_{j=k+2}^{\infty}\left[b_{1}, b_{2}, T\right]\left(f_{1}, f_{2 j}\right) x_{k}\right\|_{L^{\rho}()(w)}^{q_{\infty}}\right)^{1 / q_{\infty}} .
\end{aligned}
$$

We shall use the following estimates. If $l \leq k-1$, then pass Hölder's inequality, we have

$$
\begin{aligned}
& \int_{\mathbb{R}^{n}}\left|b_{i}(x)-b_{i}\left(y_{i}\right)\right| f_{i l} d y_{i} \\
& \leq \int_{\mathbb{R}^{n}}\left|b_{i}(x)-\left(b_{i}\right)_{B_{l}}\right| f_{i l} d y_{i}+\int_{\mathbb{R}^{n}}\left|b_{i}\left(y_{i}\right)-\left(b_{i}\right)_{B_{l}}\right| f_{i l} d y_{i} \\
& \leq\left|b_{i}(x)-\left(b_{i}\right)_{B_{l}}\right|\left\|f_{i l} \chi_{l}\right\|_{L^{p_{i}(\cdot)}\left(w_{i}\right)}\left\|\chi_{l}\right\|_{L^{p_{i}^{\prime}(\cdot)}\left(w_{i}^{-1}\right)} \\
& \quad+\left\|f_{i l} \chi_{l}\right\|_{L^{p_{i}(\cdot)}\left(w_{i}\right)}\left\|\left(b_{i}-\left(b_{i}\right)_{B_{l}}\right) \chi_{l}\right\|_{L^{p_{i}^{\prime}(\cdot)}\left(w_{i}^{-1}\right)} .
\end{aligned}
$$

By Lemmas 7 and 10, Hölder's inequality, and Definition 2 , we acquire that 


$$
\begin{aligned}
& \left\|2^{-k n} \int_{\mathbb{R}^{n}}\left|b_{i}(x)-b_{i}\left(y_{i}\right)\right| f_{i l} d y_{i} \chi_{k}\right\|_{L^{p_{i}(\cdot)}\left(w_{i}\right)} \\
& \leqslant 2^{-k n}\left\|\left(b_{i}-\left(b_{i}\right)_{B_{l}}\right) \cdot \chi_{k}\right\|_{L^{p^{(\cdot)}(w)}}\left\|f_{i l} \chi_{l}\right\|_{L^{p_{i}(\cdot)}\left(w_{i}\right)}\left\|\chi_{l}\right\|_{L^{p_{i}^{\prime}(\cdot)}\left(w_{i}^{-1}\right)}
\end{aligned}
$$

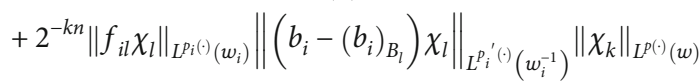

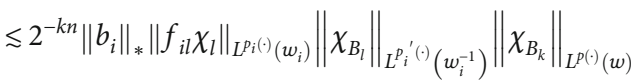

$$
\begin{aligned}
& +2^{-k n}\left\|b_{i}\right\|_{*}(k-l)\left\|f_{\mathrm{il}} \chi_{l}\right\|_{L^{p^{(\cdot)}\left(w_{i}\right)}}\left\|\chi_{B_{l}}\right\|_{L^{p_{i}^{(\cdot)}\left(w_{i}^{-1}\right)}}\left\|\chi_{B_{k}}\right\|_{L^{p^{()}(w)}} \\
& \lesssim(k-l) 2^{-k n}\left\|b_{i}\right\|_{*}\left\|f_{i l} \chi_{l}\right\|_{L^{p^{(}()}\left(w_{i}\right)}\left\|\chi_{B_{l}}\right\|_{L^{p_{i}^{\prime}(\cdot)}\left(w_{i}^{-1}\right)}\left\|\chi_{B_{k}}\right\|_{L^{P^{(\cdot)}(w)}} \\
& \lesssim(k-l) 2^{-k n}\left\|b_{i}\right\|_{*}\left\|\chi_{B_{k}}\right\|_{L_{p^{p_{i}(\cdot)}\left(w_{i}\right)}}\left\|f_{i l} w_{i} \chi_{l}\right\|_{L_{p^{\left.p^{(}\right)}}}\left\|\chi_{l} w_{i}^{-1}\right\|_{L^{p_{i}^{(}()}} \\
& \leqslant(k-l) 2^{-k n}\left\|b_{i}\right\|_{*}\left|B_{k}\right|\left\|\chi_{B_{k}}\right\|_{L^{p_{i}^{\prime}(\cdot)}\left(w_{i}^{-1}\right)}^{-1}\left\|\chi_{B_{l}}\right\|_{L_{p^{p_{i}^{\prime}(\cdot)}\left(w_{i}^{-1}\right)}}\left\|f_{i l} \chi_{l}\right\|_{L^{p_{i}(\cdot)}\left(w_{i}\right)} \\
& \leqslant(k-l) 2^{(l-k) n \delta_{i 2}}\left\|b_{i}\right\|_{*}\left\|f_{i l}\right\|_{L^{p_{i}(\cdot)}\left(w_{i}\right)} \text {. }
\end{aligned}
$$

If $l=k$, then

$$
\begin{aligned}
& \left\|2^{-k n} \int_{\mathbb{R}^{n}}\left|b_{i}(x)-b_{i}\left(y_{i}\right)\right| f_{i l} d y_{i} \chi_{k}\right\|_{L^{p_{i}(\cdot)}\left(w_{i}\right)} \\
& \lesssim 2^{-k n}\left\|\left(b_{i}-\left(b_{i}\right)_{B_{k}}\right) \cdot \chi_{k}\right\|_{L^{p^{(\cdot)}(w)}}\left\|f_{i l} \chi_{l}\right\|_{L^{p_{i}^{(\cdot)}\left(w_{i}\right)}}\left\|\chi_{l}\right\|_{L^{p_{i}^{\prime}(\cdot)}\left(w_{i}^{-1}\right)}
\end{aligned}
$$

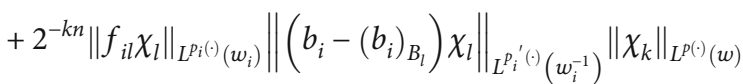

$$
\begin{aligned}
& \lesssim 2^{-k n}\left\|b_{i}\right\|_{*}\left\|\chi_{B_{k}}\right\|_{L_{L^{\left.p^{(}\right)}\left(w_{i}\right)}}\left\|f_{i l} w_{i} \chi_{l}\right\|_{L^{\left.p^{i(}\right)}}\left\|\chi_{l} w_{i}^{-1}\right\|_{L^{p_{i}^{\prime}(\cdot)}} \\
& \leqslant 2^{-k n}\left\|b_{i}\right\|_{*}\left\|\chi_{B_{k}}\right\|_{L^{p_{i}(\cdot)}\left(w_{i}\right)}\left\|\chi_{B_{l}}\right\|_{L^{p_{i}^{\prime(\cdot)}\left(w_{i}^{-1}\right)}}\left\|f_{i l} \chi_{l}\right\|_{L^{p^{p^{(}()}\left(w_{i}\right)}} \\
& \leqslant\left\|b_{i}\right\|_{*}\left\|f_{i l}\right\|_{L^{p_{i}(\cdot)}\left(w_{i}\right)} \text {. }
\end{aligned}
$$

If $l \geq k+1$, then pass Hölder's inequality, we have

$$
\begin{aligned}
& \int_{\mathbb{R}^{n}}\left|b_{i}(x)-b_{i}\left(y_{i}\right)\right| f_{i l} d y_{i} \\
& \leq \int_{\mathbb{R}^{n}}\left|b_{i}(x)-\left(b_{i}\right)_{B_{l}}\right| f_{i l} d y_{i}+\int_{\mathbb{R}^{n}}\left|b_{i}\left(y_{i}\right)-\left(b_{i}\right)_{B_{l}}\right| f_{i l} d y_{i} \\
& \leq\left|b_{i}(x)-\left(b_{i}\right)_{B_{k}}\right|\left\|f_{i l} \chi_{l}\right\|_{L^{P_{i}(\cdot)}\left(w_{i}\right)}\left\|\chi_{l}\right\|_{L^{P_{i}^{\prime}(\cdot)}\left(w_{i}^{-1}\right)} \\
& \quad+\left\|f_{i l} \chi_{l}\right\|_{L^{P_{i}(\cdot)}\left(w_{i}\right)}\left\|\left(b_{i}-\left(b_{i}\right)_{B_{k}}\right) \chi_{l}\right\|_{L^{P^{\prime}(\cdot)}\left(w_{i}^{-1}\right)} .
\end{aligned}
$$

By Lemmas 7 and 10, Hölder's inequality, and Definition 2 , we acquire that

$$
\begin{aligned}
& \left\|2^{-k n} \int_{\mathbb{R}^{n}}\left|b_{i}(x)-b_{i}\left(y_{i}\right)\right| f_{i l} d y_{i} \chi_{k}\right\|_{L^{\left.p^{(}()\right)}\left(w_{i}\right)} \\
& \lesssim 2^{-k n}\left\|\left(b_{i}-\left(b_{i}\right)_{B_{k}}\right) \cdot \chi_{k}\right\|_{L^{p^{(\cdot)}(w)}}\left\|f_{i l} \chi_{l}\right\|_{L^{p_{i}^{(\cdot)}\left(w_{i}\right)}}\left\|\chi_{l}\right\|_{L^{p_{i}^{\prime}(\cdot)}\left(w_{i}^{-1}\right)}
\end{aligned}
$$

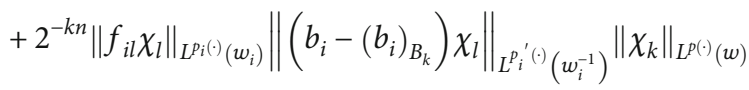

$$
\begin{aligned}
& \lesssim 2^{-k n}\left\|b_{i}\right\|_{*}\left\|f_{i l} \chi_{l}\right\|_{L^{p_{i}(\cdot)}\left(w_{i}\right)}\left\|\chi_{B_{l}}\right\|_{L^{p_{i}^{\prime}(\cdot)}\left(w_{i}^{-1}\right)}\left\|\chi_{B_{k}}\right\|_{L^{p(\cdot)}(w)} \\
& +2^{-k n}\left\|b_{i}\right\|_{*}(l-k)\left\|f_{i l} \chi_{l}\right\|_{L^{p^{(}(\cdot)}\left(w_{i}\right)}\left\|\chi_{B_{l}}\right\|_{L^{p^{p_{i}^{\prime}(\cdot)}\left(w_{i}^{-1}\right)}}\left\|\chi_{B_{k}}\right\|_{L^{p^{(\cdot)}(w)}} \\
& \lesssim 2^{-k n}(l-k)\left\|b_{i}\right\|_{*}\left\|f_{i l} \chi_{l}\right\|_{L^{p^{(\cdot)}(\cdot)}\left(w_{1}\right)}\left\|\chi_{B_{l}}\right\|_{L^{p^{p_{1}}(\cdot)}\left(w_{1}^{-1}\right)}\left\|\chi_{B_{k}}\right\|_{L^{p^{(\cdot)}(w)}} \\
& \leqslant 2^{-k n}(l-k)\left\|b_{i}\right\|_{*}\left\|\chi_{B_{k}}\right\|_{L^{p_{i}^{(\cdot)}\left(w_{i}\right)}}\left\|f_{i l} w_{i} \chi_{l}\right\|_{L^{p_{i}(\cdot)}}\left\|\chi_{l} w_{i}^{-1}\right\|_{L^{p_{i}^{\prime}(\cdot)}} \\
& \lesssim 2^{-k n}(l-k)\left\|b_{i}\right\|_{*}\left\|\chi_{B_{k}}\right\|_{L^{p^{(\cdot)}(\cdot)}\left(w_{i}\right)}\left\|\chi_{B_{l}}\right\|_{L_{L^{p^{(}(\cdot)}\left(w_{i}\right)}}\left\|\chi_{B_{l}}\right\|_{L^{p^{(\cdot)}\left(w_{i}\right)}}^{-1} \\
& \times\left\|\chi_{B_{l}}\right\|_{L_{L_{i}^{p^{\prime}(\cdot)}\left(w_{i}^{-1}\right)}}\left\|f_{i l} \chi_{l}\right\|_{L^{p_{i}^{(\cdot)}\left(w_{i}\right)}} \\
& \leqslant 2^{(l-k) n\left(1-\delta_{1 i}\right)}(l-k)\left\|b_{i}\right\|_{*}\left\|f_{i l}\right\|_{L^{p^{(\cdot)}\left(w_{i}\right)}} .
\end{aligned}
$$

By the interchange of $f_{1}$ and $f_{2}$, we see that the estimates of $E_{2}, E_{3}$, and $E_{6}$ are similar to $E_{4}, E_{7}$, and $E_{8}$, respectively. Thus, we only to estimate $E_{1}, E_{2}, E_{3}, E_{5}, E_{6}$, and $E_{9}$.

To estimate $E_{1}$, due to $l, j \leq k-2$, we infer that for $i=1,2$,

$$
\begin{aligned}
\left|x-y_{i}\right| & \geq|x|-\left|y_{i}\right|>2^{k-1}-2^{\min \{l, j\}} \\
& \geq 2^{k-2}, x \in D_{k}, y_{1} \in D_{l}, y_{2} \in D_{j} .
\end{aligned}
$$

Therefore, for $x \in D_{k}$, we have

$$
\left|K\left(x, y_{1}, y_{2}\right)\right| \leq C\left(\left|x-y_{1}\right|+\left|x-y_{2}\right|\right)^{-2 n} \leq C 2^{-2 k n} .
$$

Thus, $\forall x \in D_{k}$ and $l, j \leq k-2$, we have

$$
\begin{aligned}
& {\left[b_{1}, b_{2}, T\right]\left(f_{1 l}, f_{2 j}\right)(x)} \\
& \quad \lesssim \int_{\mathbb{R}^{2 n}} \prod_{i=1}^{2}\left|b_{i}(x)-b_{i}\left(y_{i}\right)\right|\left|K\left(x, y_{1}, y_{2}\right)\right|\left|f_{1 l}\left(y_{1}\right)\right|\left|f_{2 j}\left(y_{2}\right)\right| d y_{1} d y_{2} \\
& \quad \lesssim 2^{-2 k n} \int_{\mathbb{R}^{2 n}} \prod_{i=1}^{2}\left|b_{i}(x)-b_{i}\left(y_{i}\right)\right| f_{1 l}\left(y_{1}\right)|| f_{2 j}\left(y_{2}\right) \mid d y_{1} d y_{2} .
\end{aligned}
$$


Thus, according to Hölder's inequality, we have

$$
\begin{aligned}
& \left\|\sum_{l=-\infty}^{k-2} \sum_{j=-\infty}^{k-2}\left[b_{1}, b_{2}, T\right]\left(f_{1 l}, f_{2 j}\right) \chi_{k}\right\|_{L^{p(\cdot)}(w)} \\
& \leq \| \sum_{l=-\infty}^{k-2} 2^{-k n} \int_{\mathbb{R}^{n}}\left|b_{1}(x)-b_{1}\left(y_{1}\right)\right|\left|f_{1 l}\left(y_{1}\right)\right| d y_{1} \\
& \quad \times \sum_{j=-\infty}^{k-2} 2^{-k n} \int_{\mathbb{R}^{n}}\left|b_{2}(x)-b_{2}\left(y_{1}\right)\right|\left|f_{2 j}\left(y_{2}\right)\right| d y_{2} \chi_{k} \|_{L^{p(\cdot)}(w)} \\
& \leq\left\|\sum _ { l = - \infty } ^ { k - 2 } 2 ^ { - k n } \int _ { \mathbb { R } ^ { n } } \left|b_{1}(x)-b_{1}\left(y_{1}\right)\left\|f_{1 l}\left(y_{1}\right) \mid d y_{1} \chi_{k}\right\|_{L^{p_{1}(\cdot)}\left(w_{1}\right)}\right.\right. \\
& \quad \times\left\|\sum_{j=-\infty}^{k-2} 2^{-k n} \int_{\mathbb{R}^{n}}\left|b_{2}(x)-b_{2}\left(y_{2}\right)\right|\left|f_{2 j}\left(y_{2}\right)\right| d y_{2} \chi_{k}\right\|_{L^{p^{2}(\cdot)}\left(w_{2}\right)} .
\end{aligned}
$$

Since $1 / q(0)=1 / q_{1}(0)+1 / q_{2}(0), \lambda=\lambda_{1}+\lambda_{2}$, according to Hölder's inequality, we will have

$$
\begin{aligned}
E_{1} \lesssim & \sup _{L \leq 0, L \in \mathbb{Z}} 2^{-L \lambda}\left(\sum_{k=-\infty}^{L} 2^{k \alpha(0) q(0)}\left\|\sum_{l=-\infty}^{k-2} 2^{-k n} \int_{\mathbb{R}^{n}}\left|b_{1}(x)-b_{1}\left(y_{1}\right)\right|\left|f_{1 l}\left(y_{1}\right)\right| d y_{1} \chi_{k}\right\|_{L^{p_{1}(\cdot)}\left(w_{1}\right)}^{q(0)}\right. \\
& \left.\times\left\|\sum_{j=-\infty}^{k-2} 2^{-k n} \int_{\mathbb{R}^{n}}\left|b_{2}(x)-b_{2}\left(y_{2}\right)\right|\left|f_{2 j}\left(y_{2}\right)\right| d y_{2} \chi_{k}\right\|_{L^{p_{2}(\cdot)}\left(w_{2}\right)}^{q(0)}\right)^{1 / q(0)} \\
\leq & \sup _{L \leq 0, L \in \mathbb{Z}} 2^{-L \lambda_{1}} \\
& \times\left(\sum_{k=-\infty}^{L} 2^{k \alpha_{1}(0) q_{1}(0)}\left\|\sum_{l=-\infty}^{k-2} 2^{-k n} \int_{\mathbb{R}^{n}}\left|b_{1}(x)-b_{1}\left(y_{1}\right)\right|\left|f_{1 l}\left(y_{1}\right)\right| d y_{1} \chi_{k}\right\|_{L_{1}^{p_{1}(\cdot)}\left(w_{1}\right)}^{q_{1}(0)}\right)^{1 / q_{1}(0)} \\
& \times \sup _{L \leq 0, L \in \mathbb{Z}} 2^{-L \lambda_{2}} \\
& \times\left(\sum_{k=-\infty}^{L} 2^{k \alpha_{2}(0) q_{2}(0)}\left\|\sum_{j=-\infty}^{k-2} 2^{-k n} \int_{\mathbb{R}^{n}}\left|b_{2}(x)-b_{2}\left(y_{2}\right)\right|\left|f_{2 j}\left(y_{2}\right)\right| d y_{2} \chi_{k}\right\|_{L^{p_{2}(\cdot)}\left(w_{2}\right)}^{q_{2}(0)}\right)^{1 / q_{2}(0)} \\
:= & E_{1,1} \times E_{1,2} .
\end{aligned}
$$

where

$$
\begin{aligned}
E_{1, i}:= & \sup _{L \leq 0, L \in \mathbb{Z}} 2^{-L \lambda_{i}} \\
& \times\left\{\sum _ { k = - \infty } ^ { L } 2 ^ { k \alpha _ { i } ( 0 ) q _ { i } ( 0 ) } \left\|\sum_{l=-\infty}^{k-2} 2^{-k n} \int_{\mathbb{R}^{n}}\left|b_{i}(x)-b_{i}\left(y_{i}\right)\left\|f_{i l}\left(y_{i}\right) \mid d y_{i} \chi_{k}\right\|_{L^{p_{i}(\cdot)}\left(w_{i}\right)}^{q_{i}(0)}\right\}^{1 / q_{i}(0)} .\right.\right.
\end{aligned}
$$

Since $n \delta_{i 2}-\alpha_{i}(0)>0$, by (41) and Lemma 11, we acquire that

$$
\begin{aligned}
& E_{1, i} \leqslant \sup _{L \leq 0, L \in \mathbb{Z}} 2^{-L \lambda_{i}}\left\{\sum_{k=-\infty}^{L} 2^{k \alpha_{i}(0) q_{i}(0)}\left\|b_{i}\right\|_{*}\left(\sum_{l=-\infty}^{k-2} 2^{(l-k) n \delta_{i 2}}\left\|f_{i l}\right\|_{L^{p_{i}(\theta)}\left(w_{i}\right)}\right)^{q_{i}(0)}\right\}^{1 / q_{i}(0)} \\
& =\left\|b_{i}\right\|_{*} \sup _{L \leq 0, L \in \mathbb{Z}} 2^{-L \lambda_{i}} \times\left\{\sum_{k=-\infty}^{L}\left(\sum_{l=-\infty}^{k-2} 2^{l \alpha_{i}(0)}\left\|f_{i l}\right\|_{L^{p_{i}(i)}\left(w_{i}\right)} 2^{(l-k)\left(n \delta_{i 2}-\alpha_{i}(0)\right)}\right)^{q_{i}(0)}\right\}^{1 / q_{i}(0)} \\
& \leqslant\left\|b_{i}\right\|_{L_{L \leq 0, L \in \mathbb{Z}}} \sup 2^{-L \lambda_{i}}\left(\sum_{l=-\infty}^{L-2} 2^{l \alpha_{i}(0) q_{i}(0)}\left\|f_{i i}\right\|_{L^{p_{i}(\theta)}\left(w_{i}\right)}^{q_{i}(0)}\right)^{1 / q_{i}(0)}
\end{aligned}
$$

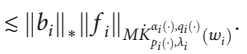

Thus, we obtain that

$$
E_{1} \lesssim\left\|f_{1}\right\|_{M \dot{K}_{p_{1}(\cdot), \lambda_{1}}^{\alpha_{1}(\cdot), q_{1}(\cdot)}\left(w_{1}\right)}\left\|f_{2}\right\|_{M \dot{K}_{p_{2}(\cdot), \lambda_{2}}^{\alpha_{2}(\cdot), q_{2}(\cdot)}\left(w_{2}\right)^{\prime}} .
$$

To estimate $E_{2}$, due to $l \leq k-2, k-1 \leq j \leq k+1$ for $i=1,2$, then, we have

$$
\left|x-y_{1}\right| \geq|x|-\left|y_{1}\right| \geq 2^{k-2}, x \in D_{k}, y_{1} \in D_{l}
$$

So, according to Hölder's inequality, we have

$$
\begin{aligned}
& \left\|\sum_{l=-\infty}^{k-2} \sum_{j=k-1}^{k+1}\left[b_{1}, b_{2}, T\right]\left(f_{1 l}, f_{2 j}\right) \chi_{k}\right\|_{L^{p(\cdot)}(w)} \\
& \leq \| \sum_{l=-\infty}^{k-2} 2^{-k n} \int_{\mathbb{R}^{n}}\left|b_{1}(x)-b_{1}\left(y_{1}\right)\right|\left|f_{1 l}\left(y_{1}\right)\right| d y_{1} \\
& \quad \times \sum_{j=k-1}^{k+1} 2^{-k n} \int_{\mathbb{R}^{n}}\left|b_{2}(x)-b_{2}\left(y_{2}\right)\right|\left|f_{2 j}\left(y_{2}\right)\right| d y_{2} \chi_{k} \|_{L^{p(\cdot)}(w)} \\
& \leq\left\|\sum_{l=-\infty}^{k-2} 2^{-k n} \int_{\mathbb{R}^{n}}\left|b_{1}(x)-b_{1}\left(y_{1}\right)\right|\left|f_{1 l}\left(y_{1}\right)\right| d y_{1} \chi_{k}\right\|_{L^{p_{1}(\cdot)}\left(w_{1}\right)} \\
& \quad \times\left\|\sum_{j=k-1}^{k+1} 2^{-k n} \int_{\mathbb{R}^{n}}\left|b_{2}(x)-b_{2}\left(y_{2}\right)\right|\left|f_{2 j}\left(y_{2}\right)\right| d y_{2} \chi_{k}\right\|_{L^{p^{(\cdot)}(\cdot)}\left(w_{2}\right)} .
\end{aligned}
$$

Since $\quad 1 / q(0)=1 / q_{1}(0)+1 / q_{2}(0), \quad \lambda=\lambda_{1}+\lambda_{2}, \quad$ by Hölder's inequality, we acquire that

$$
\begin{aligned}
E_{2} \lesssim & \sup _{L \leq 0, L \in \mathbb{Z}} 2^{-L \lambda}\left(\sum _ { k = - \infty } ^ { L } 2 ^ { k \alpha ( 0 ) q ( 0 ) } \left\|\sum _ { l = - \infty } ^ { k - 2 } 2 ^ { - k n } \int _ { \mathbb { R } ^ { n } } \left|b_{1}(x)-b_{1}\left(y_{1}\right)\left\|f_{1 l}\left(y_{1}\right) \mid d y_{1} \chi_{k}\right\|_{L^{p_{1}(\cdot)}\left(w_{1}\right)}^{q(0)}\right.\right.\right. \\
& \left.\times\left\|\sum_{j=k-1}^{k+1} 2^{-k n} \int_{\mathbb{R}^{n}}\left|b_{2}(x)-b_{2}\left(y_{2}\right)\right|\left|f_{2 j}\left(y_{2}\right)\right| d y_{2} \chi_{k}\right\|_{L^{p_{2}(\cdot)}\left(w_{2}\right)}^{q(0)}\right)^{1 / q(0)} \\
\lesssim & \sup _{L \leq 0, L \in \mathbb{Z}} 2^{-L \lambda_{1}}\left(\sum_{k=-\infty}^{L} 2^{k \alpha_{1}(0) q_{1}(0)}\right. \\
& \times\left\|\sum_{l=-\infty}^{k-2} 2^{-k n} \int_{\mathbb{R}^{n}}\left|b_{1}(x)-b_{1}\left(y_{1}\right)\left\|f_{1 l}\left(y_{1}\right) \mid d y_{1} \chi_{k}\right\|_{L^{p_{1}(\cdot)}\left(w_{1}\right)}^{q_{1}(0)}\right)^{1 / q_{1}(0)}\right. \\
& \times \sup _{L \leq 0, L \in \mathbb{Z}} 2^{-L \lambda_{2}}\left(\sum_{k=-\infty}^{L} 2^{k \alpha_{2}(0) q_{2}(0)}\right. \\
& \left.\times\left\|\sum_{j=k-1}^{k+1} 2^{-k n} \int_{\mathbb{R}^{n}}\left|b_{2}(x)-b_{2}\left(y_{2}\right)\right|\left|f_{2 j}\left(y_{2}\right)\right| d y_{2} \chi_{k}\right\|_{L^{p_{2}(\cdot)}\left(w_{2}\right)}^{q_{2}(0)}\right)^{1 / q_{2}(0)} \\
:= & E_{2,1} \times E_{2,2} .
\end{aligned}
$$

It is obviously that

$$
E_{2,1}=E_{1,1} \lesssim\left\|b_{1}\right\|_{*}\left\|f_{1}\right\|_{M \dot{K}_{p_{1}(\cdot), \lambda_{1}}^{\alpha_{1}(\cdot) q_{1}(\cdot)}\left(w_{1}\right)} .
$$

Now we estimate $E_{2,2}$. Taking (41), (42), and (44) together, we have 


$$
\begin{aligned}
& E_{2,2} \leqslant\left\|b_{2}\right\|_{L_{L \leq 0, L \in \mathbb{Z}}} \sup 2^{-L \lambda_{2}}\left(\sum_{k=-\infty}^{L} 2^{k \alpha_{2}(0) q_{2}(0)} \sum_{j=k-1}^{k+1} 2^{(j-k) n q_{2}(0)}\left\|f_{2 j} x_{j}\right\|_{L^{p^{2}(\cdot)}\left(w_{2}\right)}^{q_{2}(0)}\right)^{1 / q_{2}(0)}
\end{aligned}
$$

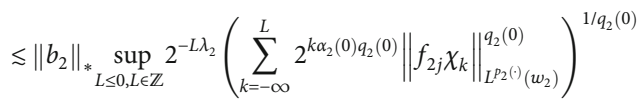

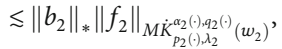

where we used $2^{-n \delta_{22}}<1$ and $2^{(j-k) n\left(1-\delta_{12}\right)}<2^{(j-k) n}<2^{2 n}$, $j \in\{k-1, k, k+1\}$ for (41) and (44), respectively. Therefore, we acquire that

$$
E_{2} \leqslant\left\|b_{1}\right\|_{*}\left\|b_{2}\right\|_{*}\left\|f_{1}\right\|_{M \dot{K}_{p_{1}(\cdot), \lambda_{1}}^{\alpha_{1}(\cdot) q_{1}(\cdot)}\left(w_{1}\right)}\left\|f_{2}\right\|_{M \dot{K}_{p_{2}}^{\alpha_{2}(\cdot), q_{2}, \cdot(\cdot)}\left(w_{2}\right)} .
$$

To estimate $E_{3}$, since $l \leq k-2, j \geq k+2$, then, we have

$$
\begin{aligned}
\left|x-y_{1}\right| & \geq|x|-\left|y_{1}\right| \geq 2^{k-2},\left|x-y_{2}\right| \geq\left|y_{2}\right|-|x| \\
& >2^{j-2}, x \in D_{k}, y_{1} \in D_{l}, y_{2} \in D_{j} .
\end{aligned}
$$

Therefore, $\forall x \in D_{k}, 1 \leq k-2, j \geq k+2$, we get

$$
\begin{aligned}
& \left|\left[b_{1}, b_{2}, T\right]\left(f_{1 l}, f_{2 j}\right)(x)\right| \\
& \quad \leq \int_{\mathbb{R}^{2 n}} \prod_{i=1}^{2}\left|b_{i}(x)-b_{i}\left(y_{i}\right)\right|\left|K\left(x, y_{1}, y_{2}\right)\right|\left|f_{1 l}\left(y_{1}\right)\right|\left|f_{2 j}\left(y_{2}\right)\right| d y_{1} d y_{2} \\
& \quad \leq 2^{-k n} 2^{-j n} \int_{\mathbb{R}^{2 n}} \prod_{i=1}^{2}\left|b_{i}(x)-b_{i}\left(y_{i}\right)\right| f_{1 l}\left(y_{1}\right)|| f_{2 j}\left(y_{2}\right) \mid d y_{1} d y_{2} .
\end{aligned}
$$

So, according to Hölder's inequality, we acquire that

$$
\begin{aligned}
& \left\|\sum_{l=-\infty}^{k-2} \sum_{j=k+2}^{\infty}\left[b_{1}, b_{2}, T\right]\left(f_{1 l}, f_{2 j}\right) \chi_{k}\right\|_{L^{p(\cdot)}(w)} \\
& \leq \| \sum_{l=-\infty}^{k-2} 2^{-k n} \int_{\mathbb{R}^{n}}\left|b_{1}(x)-b_{1}\left(y_{1}\right)\right|\left|f_{1 l}\left(y_{1}\right)\right| d y_{1} \\
& \quad \times \sum_{j=k+2}^{\infty} 2^{-j n} \int_{\mathbb{R}^{n}}\left|b_{2}(x)-b_{2}\left(y_{2}\right)\right|\left|f_{2 j}\left(y_{2}\right)\right| d y_{2} \chi_{k} \|_{L^{p(\cdot)}(w)} \\
& \leq\left\|\sum _ { l = - \infty } ^ { k - 2 } 2 ^ { - k n } \int _ { \mathbb { R } ^ { n } } \left|b_{1}(x)-b_{1}\left(y_{1}\right)\left\|f_{1 l}\left(y_{1}\right) \mid d y_{1} \chi_{k}\right\|_{L^{p_{1}(\cdot)}\left(w_{1}\right)}\right.\right. \\
& \quad \times\left\|\sum_{j=k+2}^{\infty} 2^{-j n} \int_{\mathbb{R}^{n}}\left|b_{2}(x)-b_{2}\left(y_{2}\right)\right|\left|f_{2 j}\left(y_{2}\right)\right| d y_{2} \chi_{k}\right\|_{L^{p^{2}(\cdot)}\left(w_{2}\right)} .
\end{aligned}
$$

Since $1 / q(0)=1 / q_{1}(0)+1 / q_{2}(0), \lambda=\lambda_{1}+\lambda_{2}$, according to Hölder's inequality, we acquire that

$$
\begin{aligned}
& E_{3} \lesssim \sup _{L \leq 0, L \in \mathbb{Z}} 2^{-L \lambda}\left(\sum_{k=-\infty}^{L} 2^{k \alpha(0) q(0)}\left\|\sum_{l=-\infty}^{k-2} 2^{-k n} \int_{\mathbb{R}^{n}}\left|b_{1}(x)-b_{1}\left(y_{1}\right)\right|\left|f_{1 l}\left(y_{1}\right)\right| d y_{1} \chi_{k}\right\|_{L^{P^{1}(1)}\left(w_{1}\right)}^{q(0)}\right. \\
& \left.\times\left\|\sum_{j=k+2}^{\infty} 2^{-j n} \int_{\mathbb{R}^{n}}\left|b_{2}(x)-b_{2}\left(y_{2}\right)\right|\left|f_{2 j}\left(y_{2}\right)\right| d y_{2} x_{k}\right\|_{L^{2 p^{2}(i)}\left(w_{2}\right)}^{q(0)}\right)^{1 / q(0)} \\
& \leq \sup _{L \leq 0, L \in \mathbb{Z}} 2^{-L \lambda_{1}}\left(\sum_{k=-\infty}^{L} 2^{k \alpha_{1}(0) q_{1}(0)}\right. \\
& \left.\times\left\|\sum_{l=-\infty}^{k-2} 2^{-k n} \int_{\mathbb{R}^{n}}\left|b_{1}(x)-b_{1}\left(y_{1}\right)\right|\left|f_{1 l}\left(y_{1}\right)\right| d y_{1} \chi_{k}\right\|_{L^{P_{1}}(\cdot)\left(w_{1}\right)}^{q_{1}(0)}\right)^{1 / q_{1}(0)} \\
& \times \sup _{L \leq 0, L \in \mathbb{Z}} 2^{-L \lambda_{2}}\left(\sum_{k=-\infty}^{L} 2^{k \alpha_{2}(0) q_{2}(0)}\right. \\
& \left.\times\left\|\sum_{j=k+2}^{\infty} 2^{-j n} \int_{\mathbb{R}^{n}}\left|b_{2}(x)-b_{2}\left(y_{2}\right)\right|\left|f_{2 j}\left(y_{2}\right)\right| d y_{2} \chi_{k}\right\|_{L^{2}(1)\left(w_{2}\right)}^{q_{2}(0)}\right)^{1 / q_{2}(0)} \\
& :=E_{3,1} \times E_{3,2} \text {. }
\end{aligned}
$$

It is obviously that

$$
E_{3,1}=E_{1,1} \lesssim\left\|f_{1}\right\|_{M \dot{K}_{p_{1}(\cdot), \lambda_{1}}^{\alpha_{1}(\cdot), q_{1}(\cdot)}\left(w_{1}\right)} .
$$

By (44), we acquire that

$$
\begin{aligned}
& E_{3,2} \leq\left\|b_{2}\right\|_{*} \sup _{L \leq 0, L \in \mathbb{Z}} 2^{-L \lambda_{2}} \\
& \times\left(\sum_{k=-\infty}^{L} 2^{k \alpha_{2}(0) q_{2}(0)}\left(\sum_{j=k+2}^{\infty} 2^{(k-j) n \delta_{21}}\left\|f_{2 j} \chi_{j}\right\|_{L^{p_{2}(\cdot)\left(w_{2}\right)}}\right)^{q_{2}(0)}\right)^{1 / q_{2}(0)} \\
& \leq\left\|b_{2}\right\|_{*} \sup _{L \leq 0, L \in \mathbb{Z}} 2^{-L \lambda_{2}} \\
& \times\left(\sum_{k=-\infty}^{L}\left(\sum_{j=k+2}^{L} 2^{j \alpha_{2}(0)}\left\|f_{2 j} \chi_{j}\right\|_{L^{p_{2}(\cdot)\left(w_{2}\right)}} 2^{(k-j)\left(n \delta_{21}+\alpha_{2}(0)\right)}\right)^{q_{2}(0)}\right)^{1 / q_{2}(0)} \\
& +\left\|b_{2}\right\|_{*} \sup _{L \leq 0, L \in \mathbb{Z}} 2^{-L \lambda_{2}} \\
& \times\left(\sum_{k=-\infty}^{L}\left(2^{k \alpha_{2}(0)} \sum_{j=L+1}^{0}\left\|f_{2 j} x_{j}\right\|_{L^{22}(\theta)\left(w_{2}\right)} 2^{(k-j) n \delta_{21}}\right)^{q_{2}(0)}\right)^{1 / q_{2}(0)} \\
& +\left\|b_{2}\right\|_{*} \sup _{L \leq 0, L \in \mathbb{Z}} 2^{-L \lambda_{2}} \\
& \times\left(\sum_{k=-\infty}^{L}\left(2^{k \alpha_{2}(0)} \sum_{j=1}^{\infty}\left\|f_{2 j} \chi_{j}\right\|_{L^{p_{2}(0)}\left(w_{2}\right)} 2^{(k-j) n \delta_{21}}\right)^{q_{2}(0)}\right)^{1 / q_{2}(0)} \\
& :=I_{1}+I_{2}+I_{3} \text {. }
\end{aligned}
$$

We consider $I_{1}$. By Lemma 11, we have

$$
\begin{aligned}
& I_{1} \leqslant\left\|b_{2}\right\|_{*} \sup _{L \leq 0, L \in \mathbb{Z}} 2^{-L \lambda_{2}} \\
& \times\left(\sum_{k=-\infty}^{L}\left(\sum_{j=k+2}^{L} 2^{j \alpha_{2}(0)}\left\|f_{2 j} x_{j}\right\|_{L^{p_{2}(\cdot)\left(w_{2}\right)}} 2^{(k-j)\left(n \delta_{\delta_{1}}+\alpha_{2}(0)\right)}\right)^{q_{2}(0)}\right)^{1 / q_{2}(0)} \\
& \leqslant\left\|b_{2}\right\|_{*_{L \leq 0, L \in \mathbb{Z}}} \sup 2^{-L \lambda_{2}}\left(\sum_{j=-\infty}^{L+2} 2^{j j_{2}(0) q_{2}(0)}\left\|f_{2 j} \chi_{j}\right\|_{L^{p_{2}(\cdot)}\left(w_{2}\right)}^{q_{2}(0)}\right)^{1 / q_{2}(0)} \\
& \leq\left\|b_{2}\right\|_{*}\left\|f_{2}\right\|_{M \dot{K}_{p_{2}}^{\alpha_{2}(\cdot), p_{2}(\cdot)}\left(w_{2}\right)}
\end{aligned}
$$

where $2^{-|k-j|\left(n \delta_{21}+\alpha_{2}(0)\right)}=2^{-|k-j| \alpha_{2}}$ for $\alpha_{2}=n \delta_{21}+\alpha_{2}(0)>0$. 
We consider $I_{2}$. Since $n \delta_{21}+\alpha_{2}(0)-\lambda_{2}>0$, we obtain that

$$
\begin{aligned}
& I_{2} \leq\left\|b_{2}\right\|_{*} \sup _{L \leq 0, L \in \mathbb{Z}} 2^{-L \lambda_{2}}\left(\sum _ { k = - \infty } ^ { L } \left(2^{k\left(n \delta_{21}+\alpha_{2}(0)\right)} \sum_{j=L+1}^{0} 2^{j \alpha_{2}(0)}\left\|f_{2 j} \chi_{j}\right\|_{L^{p_{2}(\cdot)}\left(w_{2}\right)}\right.\right. \\
& \left.\left.\times 2^{-j\left(n \delta_{21}+\alpha_{2}(0)\right)}\right)^{q_{2}(0)}\right)^{1 / q_{2}(0)} \\
& \leqslant\left\|b_{2}\right\|_{*} \sup _{L \leq 0, L \in \mathbb{Z}} \sup _{j \leq 0} 2^{-j \lambda_{2}} 2^{j \alpha_{2}(0)}\left\|f_{2 j} x_{j}\right\|_{L^{P^{2}(\cdot)}\left(w_{2}\right)} \\
& \times 2^{-L \lambda_{2}}\left(\sum_{k=-\infty}^{L}\left(2^{k\left(n \delta_{21}+\alpha_{2}(0)\right)} \sum_{j=L+1}^{0} 2^{-j\left(n \delta_{21}+\alpha_{2}(0)-\lambda_{2}\right)}\right)^{q_{2}(0)}\right)^{1 / q_{2}(0)} \\
& \leqslant\left\|b_{2}\right\|_{*}\left\|f_{2}\right\|_{M \dot{K}_{P_{2}(\cdot), \lambda_{2}}^{\alpha_{2}(\cdot, \cdot)}\left(w_{2}\right)} \sup _{L \leq 0, L \in \mathbb{Z}} 2^{L\left(-n \delta_{21}-\alpha_{2}(0)\right)} \\
& \cdot\left(\sum_{k=-\infty}^{L} 2^{k\left(n \delta_{21}+\alpha_{2}(0)\right) q_{2}(0)}\right)^{1 / q_{2}(0)} \\
& \leqslant\left\|b_{2}\right\|_{*}\left\|f_{2}\right\|_{M \dot{K}_{\left.p_{2}(),\right)_{2}\left(\alpha _ { 2 } \left(a_{2}(\cdot)\right.\right.}\left(w_{2}\right)} \text {. }
\end{aligned}
$$

We consider $I_{3}$. Since $n \delta_{21}+\alpha_{20}-\lambda_{2}>0$ and $n \delta_{21}$ $+\alpha_{2}(0)-\lambda_{2}>0$, we obtain

$$
\begin{aligned}
& I_{3} \leqslant\left\|b_{2}\right\|_{*} \sup _{L \leq 0, L \in \mathbb{Z}} 2^{-L \lambda_{2}}\left(\sum _ { k = - \infty } ^ { L } \left(2^{k\left(n \delta_{21}+\alpha_{2}(0)\right)}\right.\right. \\
& \left.\left.\times \sum_{j=1}^{\infty} 2^{j \alpha_{2 \infty}}\left\|f_{2 j} \chi_{j}\right\|_{L^{p_{2}(\cdot)}\left(w_{2}\right)} 2^{-j\left(n \delta_{21}+\alpha_{2 \infty}\right)}\right)^{q_{2}(0)}\right)^{1 / q_{2}(0)}
\end{aligned}
$$

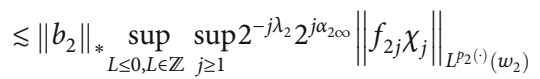

$$
\begin{aligned}
& \times 2^{-L \lambda_{2}}\left(\sum_{k=-\infty}^{L}\left(2^{k\left(n \delta_{21}+\alpha_{2}(0)\right)} \sum_{j=1}^{\infty} 2^{-j\left(n \delta_{21}+\alpha_{2 \infty}-\lambda_{2}\right)}\right)^{q_{2}(0)}\right)^{1 / q_{2}(0)} \\
& \lesssim\left\|b_{2}\right\|\left\|_{*}\right\| f_{2} \|_{M \dot{K}_{p_{2}(\cdot), \lambda_{2}}^{\alpha_{2}\left(\cdot, \alpha_{2}(\cdot)\right.}\left(w_{2}\right)} \sup _{L \leq 0, L \in \mathbb{Z}} 2^{-L \lambda_{2}}\left(\sum_{k=-\infty}^{L} 2^{k\left(n \delta_{21}+\alpha_{2}(0)\right) q_{2}(0)}\right)^{1 / q_{2}(0)} \\
& \leq\left\|b_{2}\right\|_{*}\left\|f_{2}\right\|_{M \dot{K}_{p_{2}(\cdot), \lambda_{2}}^{\alpha_{2}\left(\cdot, q_{2}(\cdot)\right.}\left(w_{2}\right)} \sup _{L \leq 0, L \in \mathbb{Z}} 2^{L\left(-\lambda_{2}+n \delta_{21}+\alpha_{2}(0)\right)} \\
& \leq\left\|b_{2}\right\|_{*}\left\|f_{2}\right\|_{M \dot{K}_{\left.p_{2}(\cdot),\right)_{2}}^{\alpha_{2}\left(\cdot, a_{2}(\cdot)\right.}\left(w_{2}\right)} \text {. }
\end{aligned}
$$

Thus, we have

$$
E_{3} \leq\left\|b_{1}\right\|_{*}\left\|b_{2}\right\|_{*}\left\|f_{1}\right\|_{M \dot{K}_{p_{1}(\cdot), \lambda_{1}}^{\alpha_{1}(\cdot) \cdot q_{1}(\cdot)}\left(w_{1}\right)}\left\|f_{2}\right\|_{M \dot{K}_{p_{2}(\cdot), \lambda_{2}}^{\alpha_{2}\left(\cdot, q_{2}(\cdot)\right.}\left(w_{2}\right)} .
$$

To estimate $E_{5}$, according to Lemma 13 and Hölder's inequality, we have

$$
\begin{aligned}
& E_{5} \lesssim \sup _{L \leq 0, L \in \mathbb{Z}} 2^{-L \lambda}\left(\sum_{k=-\infty}^{L} 2^{k \alpha(0) q(0)}\left\|\sum_{l=k-1}^{k+1} \sum_{j=k-1}^{k+1}\left[b_{1}, b_{2}, T\right]\left(f_{1}, f_{2 j}\right) \chi_{k}\right\|_{L^{(\ell)}(w)}^{q(0)}\right)^{1 / q(0)}
\end{aligned}
$$

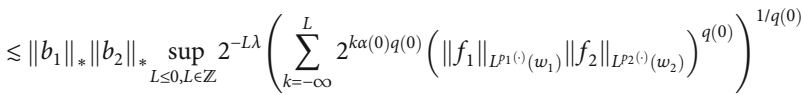

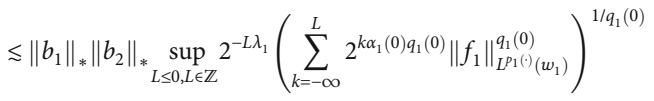

$$
\begin{aligned}
& \times 2^{-L \lambda_{2}}\left(\sum_{k=-\infty}^{L} 2^{k \alpha_{2}(0) q_{2}(0)}\left\|f_{2}\right\|_{L_{2}^{2}(0)}^{q_{2}\left(w_{2}\right)}\right)^{1 / q_{q_{2}(0)}}
\end{aligned}
$$

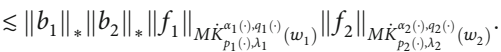

To estimate $E_{6}$, due to $k-1 \leq l \leq k+1$ and $j \geq k+2$, then we have

$$
\left|x-y_{1}\right|>2^{k-2},\left|x-y_{2}\right|>2^{j-2}, x \in D_{k}, y_{1} \in D_{l}, y_{2} \in D_{j} .
$$

Thus, $\forall x \in D_{k}, k-1 \leq 1 \leq k+1$ and $j \geq k+2$, we obtain that

$$
\begin{aligned}
& \left|\left[b_{1}, b_{2}, T\right]\left(f_{1 l}, f_{2 j}\right)(x)\right| \\
& \quad \leq \int_{\mathbb{R}^{2 n}} \prod_{i=1}^{2}\left|b_{i}(x)-b_{i}\left(y_{i}\right)\right|\left|K\left(x, y_{1}, y_{2}\right)\right|\left|f_{1 l}\left(y_{1}\right)\right|\left|f_{2 j}\left(y_{2}\right)\right| d y_{1} d y_{2} \\
& \quad \lesssim 2^{-k n} 2^{-j n} \int_{\mathbb{R}^{2 n}} \prod_{i=1}^{2}\left|b_{i}(x)-b_{i}\left(y_{i}\right)\right|\left|f_{1 l}\left(y_{1}\right)\right|\left|f_{2 j}\left(y_{2}\right)\right| d y_{1} d y_{2} .
\end{aligned}
$$

So, according to Hölder's inequality, we acquire that

$$
\begin{aligned}
& \left\|\sum_{l=k-1}^{k+1} \sum_{j=k+2}^{\infty}\left[b_{1}, b_{2}, T\right]\left(f_{1 l}, f_{2 j}\right) \chi_{k}\right\|_{L^{(\cdot)}(w)} \\
& \leq \| \sum_{l=k-1}^{k+1} 2^{-k n} \int_{\mathbb{R}^{n}}\left|b_{1}(x)-b_{1}\left(y_{1}\right)\right|\left|f_{1 l}\left(y_{1}\right)\right| d y_{1} \\
& \quad \times \sum_{j=k+2}^{\infty} 2^{-j n} \int_{\mathbb{R}^{n}}\left|b_{2}(x)-b_{2}\left(y_{2}\right)\right|\left|f_{2 j}\left(y_{2}\right)\right| d y_{2} \chi_{k} \|_{L^{p())}(w)} \\
& \leq\left\|\sum_{l=k-1}^{k+1} 2^{-k n} \int_{\mathbb{R}^{n}}\left|b_{1}(x)-b_{1}\left(y_{1}\right)\right|\left|f_{1 l}\left(y_{1}\right)\right| d y_{1} \chi_{k}\right\|_{L^{p^{(\cdot)}(\cdot)}\left(w_{1}\right)} \\
& \quad \times\left\|\sum_{j=k+2}^{\infty} 2^{-j n} \int_{\mathbb{R}^{n}}\left|b_{2}(x)-b_{2}\left(y_{2}\right)\right|\left|f_{2 j}\left(y_{2}\right)\right| d y_{2} \chi_{k}\right\|_{L^{p^{2}(\cdot)}\left(w_{2}\right)} .
\end{aligned}
$$

Since $1 / q(0)=1 / q_{1}(0)+1 / q_{2}(0), \lambda=\lambda_{1}+\lambda_{2}$, according to Hölder's inequality, we have 


$$
\begin{aligned}
& E_{6} \lesssim \sup _{L \leq 0, L \in \mathbb{Z}} 2^{-L \lambda}\left(\sum_{k=-\infty}^{L} 2^{k \alpha(0) q(0)}\left\|\sum_{l=k-1}^{k+1} 2^{-k n} \int_{\mathbb{R}^{n}}\left|b_{1}(x)-b_{1}\left(y_{1}\right)\right|\left|f_{1 l}\left(y_{1}\right)\right| d y_{1} \chi_{k}\right\|_{L^{p^{1}(1)}\left(w_{1}\right)}^{q(0)}\right. \\
& \left.\times\left\|\sum_{j=k+2}^{\infty} 2^{-j n} \int_{\mathbb{R}^{n}}\left|b_{2}(x)-b_{2}\left(y_{2}\right)\right|\left|f_{2 j}\left(y_{2}\right)\right| d y_{2} \chi_{k}\right\|_{L^{2}(\theta)\left(w_{2}\right)}^{q(0)}\right)^{1 / q(0)} \\
& \leq \sup _{L \leq 0, L \in \mathbb{Z}} 2^{-L \lambda_{1}}\left(\sum_{k=-\infty}^{L} 2^{k \alpha_{1}(0) q_{1}(0)}\right. \\
& \left.\times\left\|\sum_{l=k-1}^{k+1} 2^{-k n} \int_{\mathbb{R}^{\prime \prime}}\left|b_{1}(x)-b_{1}\left(y_{1}\right)\right|\left|f_{11}\left(y_{1}\right)\right| d y_{1} x_{k}\right\|_{L^{p^{1}(1)}\left(w_{1}\right)}^{q_{1}(0)}\right)^{1 / q_{1}(0)} \\
& \times \sup _{L \leq 0, L \in \mathbb{Z}} 2^{-L \lambda_{2}}\left(\sum_{k=-\infty}^{L} 2^{k \alpha_{2}(0) q_{2}(0)}\right. \\
& \left.\times\left\|\sum_{j=k+2}^{\infty} 2^{-j n} \int_{\mathbb{R}^{n}}\left|f_{2 j}\left(y_{2}\right)\right| d y_{2} \chi_{k}\right\|_{L^{2} \cdot(\cdot)\left(w_{2}\right)}^{q_{2}(0)}\right)^{1 / q_{2}(0)} \\
& :=E_{6,1} \times E_{6,2} \text {. }
\end{aligned}
$$

By the interchange of $f_{1}$ and $f_{2}$, we acquire that the estimate of $E_{6,1}$ is analogical to the estimate of $E_{2,2}$ and $E_{6,2}=E_{3,2}$.

To estimate $E_{9}$, since $l, j \geq k+2$, then. we get

$$
\left|x-y_{i}\right|>2^{k-2}, x \in D_{k}, y_{1} \in D_{l}, y_{2} \in D_{j} .
$$

Therefore, $\forall x \in D_{k}, l, j \geq k+2$, we have

$$
\begin{aligned}
& \left|\left[b_{1}, b_{2}, T\right]\left(f_{1 l}, f_{2 j}\right)(x)\right| \\
& \quad \leq \int_{\mathbb{R}^{2 n}} \prod_{i=1}^{2}\left|b_{i}(x)-b_{i}\left(y_{i}\right)\right|\left|K\left(x, y_{1}, y_{2}\right)\right|\left|f_{1 l}\left(y_{1}\right)\right|\left|f_{2 j}\left(y_{2}\right)\right| d y_{1} d y_{2} \\
& \quad \leq 2^{-\ln 2^{-j n}} \int_{\mathbb{R}^{2 n}} \prod_{i=1}^{2}\left|b_{i}(x)-b_{i}\left(y_{i}\right)\right|\left|f_{1 l}\left(y_{1}\right)\right|\left|f_{2 j}\left(y_{2}\right)\right| d y_{1} d y_{2} .
\end{aligned}
$$

So, according to Hölder's inequality, we obtain that

$$
\begin{aligned}
& \left\|\sum_{l=k+2}^{\infty} \sum_{j=k+2}^{\infty}\left[b_{1}, b_{2}, T\right]\left(f_{1 l}, f_{2 j}\right) \chi_{k}\right\|_{L^{p(\cdot)}(w)} \\
& \leq\left\|\sum_{l=k+2}^{\infty} 2^{-\ln } \int_{\mathbb{R}^{n}}\left|b_{1}(x)-b_{1}\left(y_{1}\right) \| f_{1 l}\left(y_{1}\right)\right| d y_{1}\right. \\
& \quad \times \sum_{j=k+2}^{\infty} 2^{-j n} \int_{\mathbb{R}^{n}}\left|b_{2}(x)-b_{2}\left(y_{2}\right)\right|\left|f_{2 j}\left(y_{2}\right)\right| d y_{2} \chi_{k} \|_{L^{p^{(\cdot)}(w)}} \\
& \quad\left\|\sum_{l=k+2}^{\infty} 2^{-\ln } \int_{\mathbb{R}^{n}}\left|b_{1}(x)-b_{1}\left(y_{1}\right)\right|\left|f_{1 l}\left(y_{1}\right)\right| d y_{1} \chi_{k}\right\|_{L^{p_{1}(\cdot)}\left(w_{1}\right)} \\
& \quad \times\left\|\sum_{j=k+2}^{\infty} 2^{-j n} \int_{\mathbb{R}^{n}}\left|b_{2}(x)-b_{2}\left(y_{2}\right)\right|\left|f_{2 j}\left(y_{2}\right)\right| d y_{2} \chi_{k}\right\|_{L^{p^{2}(\cdot)}\left(w_{2}\right)} .
\end{aligned}
$$

Since $1 / q(0)=1 / q_{1}(0)+1 / q_{2}(0), \lambda=\lambda_{1}+\lambda_{2}$, according to Hölder's inequality, we have

$$
\begin{aligned}
& E_{9} \lesssim \sup _{L \leq 0, L \in \mathbb{Z}} 2^{-L \lambda}\left(\sum_{k=-\infty}^{L} 2^{k \alpha(0) q(0)}\left\|\sum_{l=k+2}^{\infty} 2^{-\ln } \int_{\mathbb{R}^{n}}\left|b_{1}(x)-b_{1}\left(y_{1}\right)\right|\left|f_{l l}\left(y_{1}\right)\right| d y_{1} \chi_{k}\right\|_{\left.L^{1 !(}\right)\left(w_{1}\right)}^{q(0)}\right. \\
& \left.\times\left\|\sum_{j=k+2}^{\infty} 2^{-j n} \int_{\mathbb{R}^{n}}\left|b_{2}(x)-b_{2}\left(y_{2}\right)\right|\left|f_{2 j}\left(y_{2}\right)\right| d y_{2} \chi_{k}\right\|_{\left.L^{2}{ }^{2}()\right)\left(w_{2}\right)}^{q(0)}\right)^{1 / q(0)} \\
& \leq \sup _{L \leq 0, L \in \mathbb{Z}} 2^{-L \lambda_{1}}\left(\sum_{k=-\infty}^{L} 2^{k \alpha_{1}(0) q_{1}(0)}\right. \\
& \left.\times\left\|\sum_{l=k+2}^{\infty} 2^{-\ln } \int_{\mathbb{R}^{n}}\left|b_{1}(x)-b_{1}\left(y_{1}\right)\right|\left|f_{1 l}\left(y_{1}\right)\right| d y_{1} x_{k}\right\|_{L^{p_{1}(\theta)}\left(w_{1}\right)}^{q_{1}(0)}\right)^{1 / q_{1}(0)} \\
& \times \sup _{L \leq 0, L \in \mathbb{Z}} 2^{-L \lambda_{2}}\left(\sum_{k=-\infty}^{L} 2^{k \alpha_{2}(0) q_{2}(0)}\right. \\
& \left.\times\left\|\sum_{j=k+2}^{\infty} 2^{-j n} \int_{\mathbb{R}^{n}}\left|b_{2}(x)-b_{2}\left(y_{2}\right)\right|\left|f_{2 j}\left(y_{2}\right)\right| d y_{2} \chi_{k}\right\|_{L^{2} 2(x)\left(w_{2}\right)}^{q_{2}(0)}\right)^{1 / q_{2}(0)} \\
& :=E_{9,1} \times E_{9,2} \text {. }
\end{aligned}
$$

clearly, the estimate $E_{9, i}$ is analogical to the estimated $E_{3,2}$ for $i=1,2$.

Combining all the estimates of $E_{i}, i=1,2, \cdots, 9$, we obtain that

$$
E \lesssim\left\|b_{1}\right\|_{*}\left\|b_{2}\right\|_{*}\left\|f_{1}\right\|_{M \dot{K}_{p_{1}(\cdot), \lambda_{1}}^{\alpha_{1}(\cdot), q_{1}(\cdot)}\left(w_{1}\right)}\left\|f_{2}\right\|_{M \dot{K}_{p_{2}(\cdot), \lambda_{2}}^{\alpha_{2}\left(\cdot, q_{2}(\cdot)\right.}\left(w_{2}\right)} .
$$

In order to continue, we need further preparation. If $l<0$, since Proposition 6, we obtain that

$$
\begin{aligned}
& \left\|f_{i l} \chi_{l}\right\|_{L^{p^{(\cdot)}\left(w_{i}\right)}} \\
& =2^{-l \alpha_{i}(0)}\left(2^{l \alpha_{i}(0) q_{i}(0)}\left\|f_{i 1} \chi_{l}\right\|_{L^{p_{i}(\cdot)}\left(w_{i}\right)}^{q_{i}(0)}\right)^{1 / q_{i}(0)} \\
& \lesssim 2^{-l \alpha_{i}(0)}\left(\sum_{t=-\infty}^{l} 2^{t \alpha_{i}(0) q_{i}(0)}\left\|f_{i t} \chi_{t}\right\|_{L^{p_{i}(\cdot)}\left(w_{i}\right)}^{q_{i}(0)}\right)^{1 / q_{i}(0)} \\
& \leq 2^{l\left(\lambda_{i}-\alpha_{i}(0)\right)}\left(2^{-l \lambda_{i}}\left(\sum_{t=-\infty}^{l}\left\|2^{t \alpha_{i}(0)} f_{i t} \chi_{t}\right\|_{L^{p_{i}(\cdot)}\left(w_{i}\right)}^{q_{i}(0)}\right)\right)^{1 / q_{i}(0)} \\
& \leqslant 2^{l\left(\lambda_{i}-\alpha_{i}(0)\right)}\left\|f_{i}\right\|_{M \dot{K}_{p_{i}(\cdot), \lambda_{i}}^{\alpha_{i}(\cdot, \cdot)}\left(w_{i}\right)} \text {. }
\end{aligned}
$$

conclusively, we estimate $G$. according to the interchange of $f_{1}$ and $f_{2}$, we see that the estimates of $G_{2}, G_{3}$, and $G_{6}$ are similar to $G_{4}, G_{7}$, and $G_{8}$, respectively. Thus, it was only necessary to estimate $G_{1}, G_{2}, G_{3}, G_{5}, G_{6}$, and $G_{9}$. 
To estimate $G_{1}$, due to $l, j \leq k-2,1 / q_{\infty}=1 / q_{1 \infty}+1 / q_{2 \infty}$, $\lambda=\lambda_{1}+\lambda_{2}$, by (48) and Hölder's inequality, we obtain that

$$
\begin{aligned}
& G_{1} \leqslant 2^{-L \lambda}\left(\sum _ { k = 0 } ^ { L } 2 ^ { k \alpha _ { \infty } q _ { \infty } } \left\|\sum _ { l = - \infty } ^ { k - 2 } 2 ^ { - k n } \int _ { \mathbb { R } ^ { n } } \left|b_{1}(x)-b_{1}\left(y_{1}\right)\left\|f_{1 l}\left(y_{1}\right) \mid d y_{1} \chi_{k}\right\|_{L^{p}(1)\left(w_{1}\right)}^{q_{\infty}}\right.\right.\right. \\
& \left.\times\left\|\sum_{j=-\infty}^{k-2} 2^{-k n} \int_{\mathbb{R}^{n}}\left|b_{2}(x)-b_{2}\left(y_{2}\right)\right|\left|f_{2 j}\left(y_{2}\right)\right| d y_{2} \chi_{k}\right\|_{\left.L^{2}{ }^{2}()_{(2)}\right)}^{q_{\infty}}\right)^{1 / q_{\infty \infty}} \\
& \leqslant 2^{-L \lambda_{1}}\left(\sum _ { k = 0 } ^ { L } 2 ^ { k \alpha _ { 1 0 0 } q _ { 1 \infty } } \left\|\sum_{l=-\infty}^{k-2} 2^{-k n} \int_{\mathbb{R}^{n}}\left|b_{1}(x)-b_{1}\left(y_{1}\right)\left\|f_{11}\left(y_{1}\right) \mid d y_{1} \chi_{k}\right\|_{L^{1}(1)\left(w_{1}\right)}^{q_{12 \infty}}\right)^{1 / q_{12 \infty}}\right.\right. \\
& \times 2^{-L \lambda_{2}}\left(\sum_{k=0}^{L} 2^{k \alpha_{200} q_{200}}\left\|\sum_{j=-\infty}^{k-2} 2^{-k n} \int_{\mathbb{R}^{n}}\left|b_{2}(x)-b_{2}\left(y_{2}\right)\right|\left|f_{2 j}\left(y_{2}\right)\right| d y_{2} \chi_{k}\right\|_{L^{2}{ }^{2}(1)\left(w_{2}\right)}^{q_{2 \infty}}\right)^{1 / q_{20}} \\
& :=G_{1,1} \times G_{1,2} \text {. }
\end{aligned}
$$

where

$$
G_{1, i}:=2^{-L \lambda_{i}}\left(\sum _ { k = 0 } ^ { L } 2 ^ { k \alpha _ { i \infty } q _ { i \infty } } \left\|\sum_{l=-\infty}^{k-2} 2^{-k n} \int_{\mathbb{R}^{n}}\left|b_{i}(x)-b_{i}\left(y_{i}\right)\left\|f_{i l}\left(y_{i}\right) \mid d y_{i} \chi_{k}\right\|_{L^{p_{i}(\vartheta)}\left(w_{i}\right)}^{q_{i \infty \infty}}\right)^{1 / q_{i \infty 0}} .\right.\right.
$$

We consider $G_{1, i}$. By (41), we obtain that

$$
\begin{aligned}
& G_{1, i} \leq\left\|b_{i}\right\|_{*} 2^{-L \lambda_{i}}\left\{\sum _ { k = 0 } ^ { L } 2 ^ { k \alpha _ { i \infty \infty } q _ { i \infty } } \left(\sum_{l=-\infty}^{-1}\left\|f_{i l} \chi_{l}\right\|_{L^{p_{i}()()}\left(w_{i}\right)} 2^{(l-k) n \delta_{i 2}}\right.\right. \\
& \left.\left.+\sum_{l=0}^{k}\left\|f_{i l} \chi_{l}\right\|_{L^{p_{i}(\cdot)}\left(w_{i}\right)} 2^{(l-k) n \delta_{i 2}}\right)^{q_{i \infty \infty}}\right\}^{1 / q_{i \infty \infty}} \\
& \leq\left\|b_{i}\right\|_{*} 2^{-L \lambda_{i}}\left\{\sum_{k=0}^{L} 2^{k \alpha_{i \infty} q_{i \infty}}\left(\sum_{l=-\infty}^{-1}\left\|f_{i l} \chi_{l}\right\|_{\left.L^{p i(}\right)\left(w_{i}\right)} 2^{(l-k) n \delta_{i 2}}\right)^{q_{i \infty}}\right\}^{1 / q_{i \infty}} \\
& +\left\|b_{i}\right\|_{*} 2^{-L \lambda_{i}}\left\{\sum_{k=0}^{L} 2^{k \alpha_{i \infty} q_{i \infty}}\left(\sum_{l=0}^{k}\left\|f_{i l} x_{l}\right\|_{L_{i}(i)\left(w_{i}\right)} 2^{(l-k) n \delta_{i 2}}\right)^{q_{i \infty \infty}}\right\}^{1 / q_{i \infty}} \\
& :=I_{4}+I_{5} \text {. }
\end{aligned}
$$

If $q_{i \infty} \geq 1$, since $n \delta_{i 2}-\alpha_{i \infty}>0$ and $n \delta_{i 2}-\alpha_{i}(0)>0$, then by the Minkowski inequality and (79), we have

$$
\begin{aligned}
& I_{4}=\left\|b_{i}\right\|_{*} 2^{-L \lambda_{i}}\left\{\sum_{k=0}^{L} 2^{k \alpha_{i \infty \infty} q_{i \infty \infty}}\left(\sum_{l=-\infty}^{-1}\left\|f_{i l} \chi_{l}\right\|_{L^{p_{i}(\cdot)}\left(w_{i}\right)} 2^{(l-k) n \delta_{i 2}}\right)^{q_{i \infty}}\right\}^{1 / q_{i \infty \infty}} \\
& \leq\left\|b_{i}\right\|_{*} 2^{-L \lambda_{i}} \sum_{l=-\infty}^{-1}\left\|f_{i l} \chi_{l}\right\|_{L^{\left.p_{i}()\right)}\left(w_{i}\right)}\left\{\sum_{k=0}^{L}\left(2^{k \alpha_{i \infty \infty}} 2^{(l-k) n \delta_{i 2}}\right)^{q_{i \infty \infty}}\right\}^{1 / q_{i \infty}} \\
& \left.\leq\left\|b_{i}\right\|_{*} 2^{-L \lambda_{i}} \sum_{l=-\infty}^{-1} 2^{\ln \delta_{i 2}}\left\|f_{i l} \chi_{l}\right\|_{L^{p_{i}(\cdot)}\left(w_{i}\right)}\left\{\sum_{k=0}^{L} 2^{-k\left(n \delta_{i 2}-\alpha_{1 \infty}\right)}\right) q_{i 00}\right\}^{1 / q_{i \infty 0}} \\
& \leqslant\left\|b_{i}\right\|_{*}\left\|f_{i}\right\|_{\mathrm{M} \dot{K}_{P_{i}(\cdot) \lambda_{i}}^{\alpha_{i}\left(\cdot, a_{i}(\cdot)\right.}\left(w_{i}\right)} 2^{-L \lambda_{i}} \sum_{l=-\infty}^{-1} 2^{l\left(n \delta_{i_{2}}+\lambda_{i}-\alpha_{i}(0)\right)}
\end{aligned}
$$

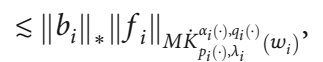

If $q_{i \infty}<1$, since $n \delta_{i 2}-\alpha_{i \infty}>0$ and $n \delta_{i 2}-\alpha_{i}(0)>0$, then by (79), we have

$$
\begin{aligned}
& I_{4} \lesssim\left\|b_{i}\right\|_{*} 2^{-L \lambda_{i}}\left(\sum_{k=0}^{L} 2^{k \alpha_{i \infty \infty} q_{i \infty}} \sum_{l=-\infty}^{-1}\left\|f_{i l} \chi_{l}\right\|_{L^{p_{i}(\cdot)}\left(w_{1}\right)^{q_{0}}}^{2^{(l-k) n \delta_{i 2} q_{i \infty 0}}}\right)^{1 / q_{i \infty 0}}
\end{aligned}
$$

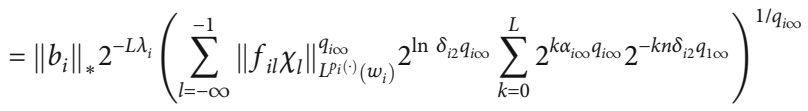

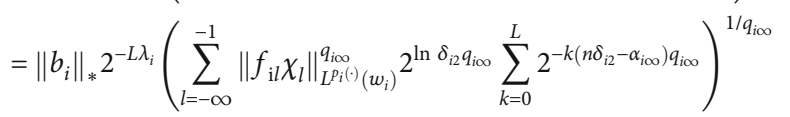

$$
\begin{aligned}
& \leq\left\|b_{i}\right\|_{*} 2^{-L \lambda_{i}}\left(\sum_{l=-\infty}^{-1}\left\|f_{i l} \chi_{l}\right\|_{L^{p_{i}()(}\left(w_{i}\right)^{q_{i \infty}}} 2^{\ln \delta_{i 2} q_{i \infty}}\right)^{1 / q_{i \infty}} \\
& \leq\left\|b_{i}\right\|_{*}\left\|f_{i}\right\|_{M K_{p_{i}(), \alpha_{i}}^{\alpha_{i}, q_{i}(i)}} 2^{-L \lambda_{i}}\left(\sum_{l=-\infty}^{-1} 2^{l\left(n \delta_{i_{12}}+\lambda_{i}-\alpha_{i}(0)\right) q_{i \infty}}\right)^{1 / q_{i \infty \infty}} \\
& \leq\left\|b_{i}\right\|_{*}\left\|f_{i}\right\|_{M \dot{K}_{\left.P_{i}()_{i}\right)()_{i}}^{a_{i}(\cdot)}}
\end{aligned}
$$

We consider $I_{5}$. Since $n \delta_{i 2}-\alpha_{i \infty}>0$, then by Lemma 11 , we have

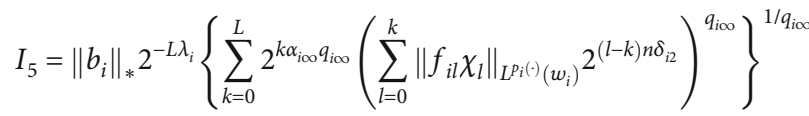

$$
\begin{aligned}
& =\left\|b_{i}\right\|_{*} 2^{-L \lambda_{i}}\left\{\sum_{k=0}^{L}\left(\sum_{l=0}^{k} 2^{l \alpha_{i \infty}}\left\|f_{i l} \chi_{l}\right\|_{L^{p_{i}(\cdot)}\left(w_{i}\right)} 2^{(l-k)\left(n \delta_{i 2}-\alpha_{i \infty \infty}\right)}\right)^{q_{i \infty \infty}}\right\}^{1 / q_{i \infty}} \\
& \leq\left\|b_{i}\right\|_{*} 2^{-L \lambda_{i}}\left(\sum_{l=0}^{k} 2^{l \alpha_{i \infty} q_{i \infty}}\left\|f_{i l} \chi_{l}\right\|_{L^{p^{i}(\cdot)}\left(w_{i}\right)}^{q_{i \infty}}\right)^{1 / q_{i \infty}} \\
& \leq\left\|b_{i}\right\|_{*}\left\|f_{i}\right\|_{M \dot{K}_{p_{i}(\cdot), i_{i}\left(\alpha _ { i } \left(a_{i}(\cdot)\right.\right.}\left(w_{i}\right)}
\end{aligned}
$$

where we wrote $2^{-|k-l|\left(n \delta_{i 2}-\alpha_{i \infty}\right)} \lesssim 2^{-|k-l| \alpha_{i}}$ for $\alpha_{i}=n \delta_{i 2}-\alpha_{i \infty}$.

Thus, we get

$$
G_{1} \lesssim\left\|b_{i}\right\|_{*}\left\|b_{2}\right\|_{*}\left\|f_{1}\right\|_{M \dot{K}_{p_{1}(\cdot), \lambda_{1}}^{\alpha_{1}(\cdot), q_{1}(\cdot)}\left(w_{1}\right)}\left\|f_{2}\right\|_{M \dot{K}_{p_{2}}^{\alpha_{2}(\cdot), \lambda_{2}, \lambda_{2}(\cdot)}\left(w_{2}\right)} .
$$

To estimate $G_{2}$, due to $l \leq k-2, k-1 \leq j \leq k+1$, $1 / q_{\infty}=1 / q_{1 \infty}+1 / q_{2 \infty}, \lambda=\lambda_{1}+\lambda_{2}$, by (54) and Hölder's inequality, we obtain that

$$
\begin{aligned}
& G_{2} \leqslant 2^{-L \lambda}\left(\sum_{k=0}^{L} 2^{k \alpha_{\infty} q_{\infty}}\left\|\sum_{l=-\infty}^{k-2} 2^{-k n} \int_{\mathbb{R}^{n}}\left|b_{1}(x)-b_{1}\left(y_{1}\right)\right|\left|f_{11}\left(y_{1}\right)\right| d y_{1} \chi_{k}\right\|_{L^{1}{ }^{1}(1)\left(w_{1}\right)}^{q_{\infty}}\right. \\
& \left.\times\left\|\sum_{j=k-1}^{k+1} 2^{-k n} \int_{\mathbb{R}^{n}}\left|b_{2}(\mathrm{x})-b_{2}\left(y_{2}\right)\right|\left|f_{2 j}\left(y_{2}\right)\right| d y_{2} \chi_{k}\right\|_{L^{2}{ }^{2}\left(w^{\prime}\left(w_{2}\right)\right.}^{q_{\infty}}\right)^{1 / q_{\infty}} \\
& \leqslant 2^{-L \lambda_{1}}\left(\sum_{k=0}^{L} 2^{k k_{100} q_{100}}\left\|\sum_{l=-\infty}^{k-2} 2^{-k n} \int_{\mathbb{R}^{n}}\left|b_{1}(x)-b_{1}\left(y_{1}\right)\right|\left|f_{11}\left(y_{1}\right)\right| d y_{1} x_{k}\right\|_{L^{p_{1}(1)}\left(w_{1}\right)}^{q_{100}}\right)^{1 / q_{1 \infty}} \\
& \times 2^{-L \lambda_{2}}\left(\sum_{k=0}^{L} 2^{k \alpha_{20} q_{200}}\left\|\sum_{j=k-1}^{k+1} 2^{-k \mathrm{n}} \int_{\mathbb{R}^{n}}\left|b_{2}(x)-b_{2}\left(y_{2}\right)\right|\left|f_{2 j}\left(y_{2}\right)\right| d y_{2} \chi_{k}\right\|_{L^{2}(\theta)\left(w_{2}\right)}^{q_{200}}\right)^{1 / q_{200}} \\
& :=G_{2,1} \times G_{2,2} \text {. }
\end{aligned}
$$

It is obviously that

$$
G_{2,1}=G_{1,1} \lesssim\left\|b_{1}\right\|_{*}\left\|f_{1}\right\|_{M \dot{K}_{p_{1}(\cdot), \lambda_{1}}^{\alpha_{1}(\cdot), \alpha_{1}(\cdot)}\left(w_{1}\right)} .
$$


Now, we estimate $G_{2,2}$. Combing (41), (42), and (44), we have

$$
\begin{aligned}
G_{2,2} & \leqslant\left\|b_{2}\right\|_{*} 2^{-L \lambda_{2}}\left(\sum_{k=0}^{L} 2^{k \alpha_{2 \infty} q_{2 \infty}} \sum_{j=k-1}^{k+1} 2^{(j-k) n q_{2 \infty}}\left\|f_{2 j} \chi_{j}\right\|_{L^{p^{2}(\cdot)}\left(w_{2}\right)}^{q_{2 \infty}}\right)^{1 / q_{2 \infty}} \\
& \leq\left\|b_{2}\right\|_{*} 2^{-L \lambda_{2}}\left(\sum_{k=0}^{L} 2^{k \alpha_{2 \infty} q_{2 \infty}}\left\|f_{2} \chi_{k}\right\|_{L^{2 \infty}(\cdot)\left(w_{2}\right)}^{q_{2 \infty}}\right)^{1 / q_{2 \infty}} \\
& \leq\left\|b_{2}\right\|_{*}\left\|f_{2}\right\|_{M \dot{K}_{\left.p_{2}(\cdot),\right)_{2}}^{\alpha_{2}(\cdot) \cdot(\cdot)}\left(w_{2}\right)}
\end{aligned}
$$

where we used $2^{-n \delta_{22}}<1$ and $2^{(j-k) n\left(1-\delta_{12}\right)}<2^{(j-k) n}$ for (41) and (44), respectively.

Thus, we acquire that

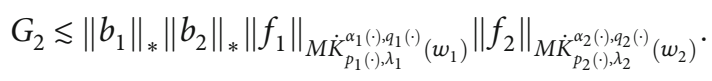

To estimate $G_{3}$, since $l \leq k-2, j \geq k+2,1 / q_{\infty}=1 / q_{1 \infty}+$ $1 / q_{2 \infty}, \lambda=\lambda_{1}+\lambda_{2}$, using (61) and Hölder's inequality, we obtain that

$$
\begin{aligned}
& G_{3} \leqslant 2^{-L \lambda}\left(\sum_{k=0}^{L} 2^{k \alpha_{\infty} q_{\infty}}\left\|\sum_{l=-\infty}^{k-2} 2^{-k n} \int_{\mathbb{R}^{n}}\left|b_{1}(x)-b_{1}\left(y_{1}\right)\right|\left|f_{1 l}\left(y_{1}\right)\right| d y_{1} \chi_{k}\right\|_{L^{1 ! l}(t)\left(w_{1}\right)}^{q_{\infty}}\right. \\
& \left.\times \|\left.\sum_{j=k+2}^{\infty} 2^{-j n} \int_{\mathbb{R}^{n}}\left|b_{2}(x)-b_{2}\left(y_{2}\right)\right|\left|f_{2 j}\left(y_{2}\right)\right| d y_{2} \chi_{k}\right|_{L^{2}{ }^{2}(1)\left(w_{2}\right)} ^{q_{\infty}}\right)^{1 / q_{q_{\infty}}} \\
& \leqslant 2^{-L \lambda_{1}}\left(\sum _ { k = 0 } ^ { L } 2 ^ { k \alpha _ { 1 0 } q _ { 1 \infty } } \left\|\sum_{l=-\infty}^{k-2} 2^{-k n} \int_{\mathbb{R}^{n}}\left|b_{1}(x)-b_{1}\left(y_{1}\right)\left\|f_{11}\left(y_{1}\right) \mid d y_{1} \chi_{k}\right\|_{L^{1}(\theta)\left(w_{1}\right)}^{q_{1 \infty}}\right)^{1 / q_{100}}\right.\right. \\
& \times 2^{-L \lambda_{2}}\left(\sum_{k=0}^{L} 2^{k k_{20} q_{2 \infty}}\left\|\sum_{j=k+2}^{\infty} 2^{-j n} \int_{\mathbb{R}^{n}}\left|b_{2}(x)-b_{2}\left(y_{2}\right)\right|\left|f_{2 j}\left(y_{2}\right)\right| d y_{2} \chi_{k}\right\|_{L^{2}{ }^{2 \cdot(2)}\left(w_{2}\right)}^{\left.q_{2 \infty}\right)}\right)^{1 / q_{2 \infty}} \\
& :=G_{3,1} \times G_{3,2} \text {. }
\end{aligned}
$$

It is obviously that

$$
G_{3,1}=G_{1,1} \lesssim\left\|b_{1}\right\|_{*}\left\|f_{1}\right\|_{M \dot{K}_{p_{1}(\cdot), \lambda_{1}}^{\alpha_{1}\left(\cdot, q_{1}(\cdot)\right.}\left(w_{1}\right)} .
$$

Since $n \delta_{12}+\alpha_{2 \infty}>0$, by (44) and Lemma 11, we obtain that

$$
\begin{aligned}
G_{3,2} \leqslant & \left\|b_{2}\right\|_{*} 2^{-L \lambda_{2}}\left(\sum_{k=0}^{L} 2^{k \alpha_{2 \infty} q_{2 \infty}}\left(\sum_{j=k+2}^{\infty} 2^{(k-j) n \delta_{21}}\left\|f_{2 j} x_{j}\right\|_{L^{p_{2}(\cdot)}\left(w_{2}\right)}\right)^{q_{2 \infty}}\right)^{1 / q_{2 \infty}} \\
\lesssim & \left\|b_{2}\right\|_{*} 2^{-L \lambda_{2}}\left(\sum_{k=0}^{L}\left(\sum_{j=k+2}^{L+2} 2^{j \alpha_{2 \infty}}\left\|f_{2 j} x_{j}\right\|_{L^{p_{2}(\cdot)}\left(w_{2}\right)} 2^{(k-j)\left(n \delta_{21}+\alpha_{2 \infty}\right)}\right)^{q_{2 \infty}}\right)^{1 / q_{2 \infty}} \\
& +\left\|b_{2}\right\|_{*} 2^{-L \lambda_{2}}\left(\sum_{k=0}^{L}\left(2^{k \alpha_{2 \infty}} \sum_{j=L+3}^{\infty}\left\|f_{2 j} x_{j}\right\|_{L^{p_{2}(\cdot)}\left(w_{2}\right)} 2^{(k-j) n \delta_{21}}\right)^{q_{2 \infty}}\right)^{1 / q_{2 \infty}} \\
= & I_{6}+I_{7} .
\end{aligned}
$$

We consider $I_{6}$. By Lemma 11, we obtain that

$$
\begin{aligned}
& I_{6} \lesssim\left\|b_{2}\right\|_{*}{ }^{-L \lambda_{2}}\left(\sum_{k=0}^{L}\left(\sum_{j=k+2}^{L+2} 2^{j \alpha_{2 \infty}}\left\|f_{2 j} \chi_{j}\right\|_{L^{p 2(\cdot)}\left(w_{2}\right)} 2^{(k-j)\left(n \delta_{21}+\alpha_{2 \infty}\right)}\right)^{q_{2 \infty}}\right)^{1 / q_{2 \infty}} \\
& \leq\left\|b_{2}\right\|_{*} 2^{-L \lambda_{2}}\left(\sum_{j=0}^{L+2} 2^{j \alpha_{2 \infty} q_{2 \infty}}\left\|f_{2 j} \chi_{j}\right\|_{L^{p_{2}(\cdot)\left(w_{2}\right)}}^{q_{2 \infty}}\right)^{1 / q_{2 \infty}}
\end{aligned}
$$

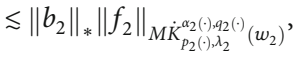

where wrote $2^{-|k-j|\left(n \delta_{21}+\alpha_{2 \infty}\right)}=2^{-|k-j| \zeta_{2}}$ for $\zeta_{2}=n \delta_{21}+\alpha_{2 \infty}$ $>0$.

We consider $I_{7}$. Since $n \delta_{21}+\alpha_{2 \infty}-\lambda_{2}>0$, we have

$$
\begin{aligned}
& I_{7} \leqslant\left\|b_{2}\right\|_{*}
\end{aligned}
$$

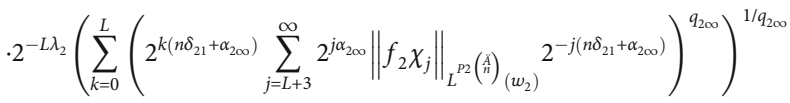

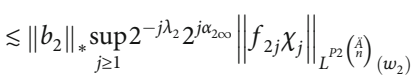

$$
\begin{aligned}
& \times 2^{-L \lambda_{2}}\left(\sum_{k=0}^{L}\left(2^{k\left(n \delta_{21}+\alpha_{2 \infty}\right)} \sum_{j=L+3}^{\infty} 2^{-j\left(n \delta_{12}+\alpha_{2 \infty}-\lambda_{2}\right)}\right)^{q_{2 \infty}}\right)^{1 / q_{2 \infty}}
\end{aligned}
$$

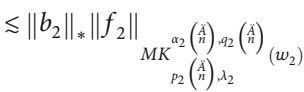

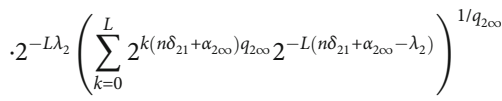

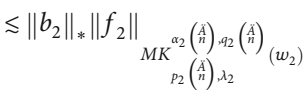

$$
\begin{aligned}
& \cdot 2^{-L \lambda_{2} 2^{\left(n \delta_{21}+\alpha_{2 \infty}\right) L} 2^{-L\left(n \delta_{21}+\alpha_{2 \infty}-\lambda_{2}\right)}}
\end{aligned}
$$

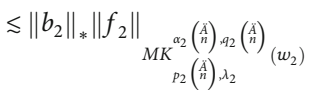

Thus, we get

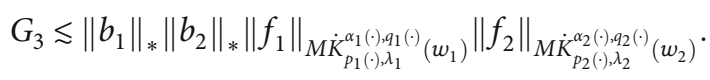

To estimate $G_{5}$, according to Lemma 13 and Hölder's inequality, we have

$$
\begin{aligned}
& G_{5} \lesssim 2^{-L \lambda}\left(\sum_{k=0}^{L} 2^{k \alpha_{\infty} q_{\infty}}\left\|\sum_{l=k-1}^{k+1} \sum_{j=k-1}^{k+1}\left[b_{1}, b_{2}, T\right]\left(f_{1 l}, f_{2 j}\right) \chi_{k}\right\|_{L^{p())}(w)}^{q_{\infty}}\right)^{1 / q_{\infty}} \\
& \leqslant 2^{-L \lambda}\left(\sum_{k=0}^{L} 2^{k \alpha_{\infty} q_{\infty}}\left(\left\|b_{1}\right\|_{*}\left\|b_{2}\right\|_{*}\left\|f_{1}\right\|_{L^{p_{1}(\cdot)}\left(w_{1}\right)}\left\|f_{2}\right\|_{L^{p_{2}(\cdot)}\left(w_{2}\right)}\right)^{q_{\infty}}\right)^{1 / q_{\infty}} \\
& \leq\left\|b_{1}\right\|_{*} 2^{-L \lambda_{1}}\left(\sum_{k=0}^{L} 2^{k \alpha_{10} q_{100}}\left\|f_{1}\right\|_{L^{p}(\cdot)\left(w_{1}\right)}^{q_{10}}\right)^{1 / q_{100}} \\
& \times\left\|b_{2}\right\|_{*} 2^{-L \lambda_{2}}\left(\sum_{k=0}^{L} 2^{k \alpha_{2 \infty} q_{2 \infty}}\left\|f_{2}\right\|_{L^{2(-)}\left(w_{2}\right)}^{q_{2 \infty}}\right)^{1 / q_{2 \infty}}
\end{aligned}
$$

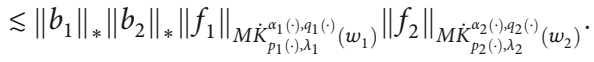


To estimate $G_{6}$, due to $k-1 \leq l \leq k+1$ and $j \geq k+2$, $1 / q_{\infty}=1 / q_{1 \infty}+1 / q_{2 \infty}, \lambda=\lambda_{1}+\lambda_{2}$, using (72) and Hölder's inequality, we obtain that

$$
\begin{aligned}
G_{6} \leqslant & 2^{-L \lambda}\left(\sum_{k=0}^{L} 2^{k \alpha_{\infty} q_{\infty}}\left\|\sum_{l=k-1}^{k+1} 2^{-k n} \int_{\mathbb{R}^{n}}\left|b_{1}(x)-b_{1}\left(y_{1}\right)\right|\left|f_{1 l}\left(y_{1}\right)\right| d y_{1} \chi_{k}\right\|_{L^{P_{1}(\cdot)}\left(w_{1}\right)}^{q_{\infty}}\right. \\
& \left.\times\left\|\sum_{j=k+2}^{\infty} 2^{-j n} \int_{\mathbb{R}^{n}}\left|b_{2}(x)-b_{2}\left(y_{2}\right)\right|\left|f_{2 j}\left(y_{2}\right)\right| d y_{2} \chi_{k}\right\|_{L^{p_{2}(\cdot)}\left(w_{2}\right)}^{q_{\infty}}\right)^{1 / q_{\infty}} \\
\leqslant & 2^{-L \lambda_{1}}\left(\sum _ { k = 0 } ^ { L } 2 ^ { k \alpha _ { 1 \infty \infty } q _ { 1 \infty } } \left\|\sum_{l=k-1}^{k+1} 2^{-k n} \int_{\mathbb{R}^{n}}\left|b_{1}(x)-b_{1}\left(y_{1}\right)\left\|f_{1 l}\left(y_{1}\right) \mid d y_{1} \chi_{k}\right\|_{L^{p_{1}(\cdot)}\left(w_{1}\right)}^{q_{1 \infty}}\right)^{1 / q_{1 \infty}}\right.\right. \\
& \times 2^{-L \lambda_{2}}\left(\sum_{k=0}^{L} 2^{k \alpha_{2 \infty} q_{2 \infty}}\left\|\sum_{j=k+2}^{\infty} 2^{-j n} \int_{\mathbb{R}^{n}}\left|b_{2}(x)-b_{2}\left(y_{2}\right)\right|\left|f_{2 j}\left(y_{2}\right)\right| d y_{2} \chi_{k}\right\|_{L^{p_{2}(\cdot)}\left(w_{2}\right)}^{q_{2 \infty}}\right)^{1 / q_{2 \infty}} \\
:= & G_{6,1} \times G_{6,2} .
\end{aligned}
$$

Since the symmetry of $f_{1}$ and $f_{2}$, we can know that the estimate $G_{6,1}$ is analogical to the estimated $G_{1,2}$ and $G_{6,2}=G_{3,2}$.

To estimate $G_{9}$, due to $l, j \geq k+2,1 / q_{\infty}=1 / q_{1 \infty}+1 / q_{2 \infty}$, $\lambda=\lambda_{1}+\lambda_{2}$, using (76) and Hölder's inequality, we obtain that

$$
\begin{aligned}
G_{9} \leqslant & 2^{-L \lambda}\left(\sum_{k=0}^{L} 2^{k \alpha_{\infty} q_{\infty}}\left\|\sum_{l=k+2}^{\infty} 2^{-\ln } \int_{\mathbb{R}^{n}}\left|b_{1}(x)-b_{1}\left(y_{1}\right)\right|\left|f_{1 l}\left(y_{1}\right)\right| d y_{1} \chi_{k}\right\|_{L^{p_{1}(\cdot)}\left(w_{1}\right)}^{q_{\infty}}\right. \\
& \left.\times\left\|\sum_{j=k+2}^{\infty} 2^{-j n} \int_{\mathbb{R}^{n}}\left|b_{2}(x)-b_{2}\left(y_{2}\right)\right|\left|f_{2 j}\left(y_{2}\right)\right| d y_{2} \chi_{k}\right\|_{L^{p_{2}(\cdot)\left(w_{2}\right)}}^{q_{\infty}}\right)^{1 / q_{\infty}} \\
\leqslant & 2^{-L \lambda_{1}}\left(\sum _ { k = 0 } ^ { L } 2 ^ { k \alpha _ { 1 \infty } q _ { 1 \infty \infty } } \left\|\sum_{l=k+2}^{\infty} 2^{-\ln } \int_{\mathbb{R}^{n}}\left|b_{1}(x)-b_{1}\left(y_{1}\right)\left\|f_{1 l}\left(y_{1}\right) \mid d y_{1} \chi_{k}\right\|_{L^{p_{1}(\cdot)}\left(w_{1}\right)}^{q_{1 \infty}}\right)^{1 / q_{1 \infty}}\right.\right. \\
& \times 2^{-L \lambda_{2}}\left(\sum_{k=0}^{L} 2^{k \alpha_{2 \infty} q_{2 \infty}}\left\|\sum_{j=k+2}^{\infty} 2^{-j n} \int_{\mathbb{R}^{n}}\left|b_{2}(x)-b_{2}\left(y_{2}\right)\right|\left|f_{2 j}\left(y_{2}\right)\right| d y_{2} \chi_{k}\right\|_{L^{p_{2}(\cdot)}\left(w_{2}\right)}^{q_{2 \infty}}\right)^{1 / q_{2 \infty}} \\
:= & G_{9,1} \times G_{9,2} .
\end{aligned}
$$

clearly, the estimate $G_{9, i}$ is analogical to the estimated $G_{3,2}$ for $i=1,2$.

Combining all the estimates ofr $G_{i}$ together, $i=1,2, \cdots, 9$, we obtain that

$$
G \lesssim\left\|f_{1}\right\|_{M \dot{K}_{p_{1}(\cdot), \lambda_{1}}^{\alpha_{1}(\cdot), q_{1}(\cdot)}\left(w_{1}\right)}\left\|f_{2}\right\|_{M \dot{K}_{p_{2}(\cdot), \lambda_{2}}^{\alpha_{2}(\cdot), q_{2}(\cdot)}\left(w_{2}\right)} .
$$

Combining the above estimates for $E, F$, and $G$, the proof of Theorem 8 is completed.

\section{Data Availability}

No data were used to support this study.

\section{Conflicts of Interest}

The authors declare that they have no competing interests.

\section{Authors' Contributions}

All authors contributed equally and significantly in writing this paper. All authors read and approved the final manuscript.

\section{Acknowledgments}

The work is partially supported by the National Natural Science Foundation of China (Grant No. 11871184).

\section{References}

[1] A. W. Huang and J. S. Xu, "Multilinear singular integrals and commutators in variable exponent Lebesgue spaces," Applied Mathematics: A Journal of Chinese Universities(Series B), vol. 25, no. 1, article 2167, pp. 69-77, 2010.

[2] Y. Hu, Y. X. He, and Y. S. Wang, "The commutators of fractional integrals on generalized Herz spaces," Journal of Function Spaces, vol. 2014, Article ID 428493, 6 pages, 2014.

[3] C. Q. Tang, Q. Wu, and J. S. Xu, "Commutators of multilinear Calderón-Zygmund operator and BMO functions in HerzMorrey spaces with variable exponents," Journal of Function Spaces, vol. 2014, Article ID 162518, 12 pages, 2014.

[4] D. Chen, S. Lu, and S. Mao, "Multiple weighted estimates for maximal vector-valued commutator of multilinear CalderónZygmund singular integrals," Frontiers of mathematics in China, vol. 12, no. 3, pp. 531-558, 2017.

[5] H. B. Wang, J. S. Xu, and J. Tan, "Boundedness of multilinear singular integrals on central Morrey spaces with variable exponents," Frontiers of Mathematics in China, vol. 15, no. 5, pp. 1011-1034, 2020.

[6] D. Cruz-Uribe, A. Fiorenza, J. M. Martell, and C. Pérez, "The boundedness of classical operators on variable $\mathrm{L}^{\mathrm{P}}$ spaces," Annales-Academice Scientiarum Fennicee Mathematica, vol. 31, no. 1, pp. 239-264, 2006.

[7] D. Cruz-Uribe, SFO, A. Fiorenza, and C. J. Neugebauer, "Weighted norm inequalities for the maximal operator on variable Lebesgue spaces," Journal of Mathematical Analysis and Applications, vol. 394, no. 2, pp. 744-760, 2012.

[8] B. Muckenhoupt, "Weighted norm inequalities for the hardy maximal function," Transactions of the American Mathematical Society, vol. 165, pp. 207-226, 1972.

[9] D. Cruz-Uribe, L. Diening, and P. Hästö, “The maximal operator on weighted variable Lebesgue spaces," Fractional Calculus and Applied Analysis, vol. 14, no. 3, pp. 361-374, 2011.

[10] D. Cruz-Uribe and L. A. Wang, "Extrapolation and weighted norm inequalities in the variable Lebesgue spaces," Transactions of the American Mathematical Society, vol. 369, no. 2, pp. 1205-1235, 2017.

[11] M. Izuki, "Remarks on Muckenhoupt weights with variable exponent," Journal of Analysis and Applications, vol. 11, no. 1, pp. 27-41, 2013.

[12] M. Izuki, E. Nakai, and Y. Sawano, "Wavelet characterization and modular inequalities for weighted Lebesgue spaces with variable exponent," Annales Academice Scientiarum Fennica, vol. 40, no. 2, pp. 551-571, 2015.

[13] S. R. Wang and J. S. Xu, "Weighted norm inequality for bilinear Calderón-Zygmund operators on Herz-Morrey spaces with variable exponents," Journal of Inequalities and Applications, vol. 2019, no. 1, Article ID 251, 2019. 
[14] M. Izuki and T. Noi, "Boundedness of fractional integrals on weighted Herz spaces with variable exponent," Journal of Inequalities and Applications, vol. 2016, no. 1, Article ID 199, 2016.

[15] M. Izuki and T. Noi, "An intrinsic square function on weighted Herz spaces with variable exponent," Journal of Mathematical Inequalities, vol. 11, no. 3, pp. 799-816, 2016.

[16] S. R. Wang and J. S. Xu, "Commutators of bilinear hardy operators on weighted Herz-Morrey spaces with variable exponents," Acta Mathematica Sinica(Chinese Series), vol. 64, no. 1, pp. 123-138, 2021.

[17] Y. Sawano, Theory of Besov Spaces, vol. 56, Springer, Singapore, 2018. 